\title{
Three-dimensional P- and S-wave velocity profiling of geotechnical sites using full-waveform inversion driven by field data
}

\author{
Arash Fathi $^{\mathrm{a}}$, Babak Poursartip ${ }^{\mathrm{b}}$, Kenneth H. Stokoe II ${ }^{\mathrm{b}}$, Loukas F. Kallivokas ${ }^{\mathrm{a}, \mathrm{b}, *}$ \\ ${ }^{a}$ Institute for Computational Engineering and Sciences \\ ${ }^{b}$ Department of Civil, Architectural and Environmental Engineering \\ The University of Texas at Austin, Austin, TX, USA
}

\begin{abstract}
We discuss the application of a recently developed full-waveform-inversion-based technique to the imaging of geotechnical sites using field-collected data. Specifically, we address the profiling of arbitrarily heterogeneous sites in terms of $\mathrm{P}$ - and S-wave velocities in three dimensions, using elastic waves as probing agents. We cast the problem of finding the spacial distribution of the elastic soil properties as an inverse medium problem, directly in the time domain, and use perfectly-matched-layers (PMLs) to account for the semi-infinite extent of the site under investigation.

After briefly reviewing the theoretical and computational aspects of the employed technique, we focus on the characterization of the George E. Brown Jr. Network for Earthquake Engineering Simulation site in Garner Valley, California (NEES@UCSB). We compare the profiles obtained from our full-waveform-inversion-based methodology against the profiling obtained from the Spectral-Analysis-of-Surface-Waves (SASW) method, and report agreement. In an attempt to validate our methodology, we also compare the recorded field data at select control sensors that were not used for the full-waveform inversion, against the response at the same sensors, computed based on the full-waveform-inverted profiles. We report very good agreement at the control sensors, which is a strong indicator of the correctness of the inverted profiles. Overall, the systematic framework discussed herein seems robust, general, practical, and promising for three-dimensional site characterization purposes.
\end{abstract}

Keywords: Site characterization, Full-waveform inversion (FWI), Elastic waves, Perfectly-matched-layers (PMLs), Spectral-Analysis-of-Surface-Waves (SASW), Vibroseis, Field data processing

\footnotetext{
${ }^{*}$ Corresponding author.

Email addresses: arash.fathi@utexas.edu (Arash Fathi), babakp@utexas.edu (Babak Poursartip), k.stokoe@mail.utexas.edu (Kenneth H. Stokoe II), loukas@mail.utexas.edu (Loukas F. Kallivokas)
} 


\section{Introduction}

Reliable three-dimensional P- and S-wave velocity profiles of the near-surface soil have great value in geotechnical engineering practice, and can play a vital role in the safe design of critical components of the civil infrastructure, such as nuclear power plants, bridges, and hospitals, among others. Wave velocity profiling of the soil is referred to as seismic site characterization. Seismic site characterization techniques can be broadly classified into two categories: invasive and non-invasive. Invasive techniques, such as borehole-based methods, are costly and can typically provide only localized information of the site under investigation, while, non-invasive techniques are capable of providing global imaging, and are generally more cost-efficient. Non-invasive profiling of the soil shares common elements with medical imaging [17]. In both cases, elastic waves are used to interrogate the unseeable medium, and the medium's response to this probing is subsequently used for driving the imaging. In the mathematical sciences, this process is referred to as solving an inverse medium problem.

Our goal is to find the distribution of the $\mathrm{P}$-wave velocity $c_{p}(\mathbf{x})$, and $\mathrm{S}$-wave velocity $c_{s}(\mathbf{x})$ of the soil in three-space dimensions ${ }^{1}$. We use a seismic vibrator to apply loads on the ground surface, which results in the propagation of elastic waves in the soil. Due to the possibly heterogeneous character of the site, multiple reflections and refractions occur. The time-history response of the soil medium to this loading is recorded by using geophones, spatially arranged over the formation's surface, and is, hereafter, referred to as measured response (Fig. 1(a)). Arriving at a material profile can then be achieved by minimizing the difference between the measured response at sensor locations, and a computed response corresponding to a trial distribution of the material parameters, under the same loading, using a computational model. The computational model should mimic the physics of the problem as closely as possible. A key challenge is proper termination of the semi-infinite extent of the domain of interest ${ }^{2}$. We use perfectly-matched-layers (PMLs) for domain truncation (Fig. 1(b)) [5, 15, 39], since they are among the best available tools in the presence of heterogeneity [15]. A PML is a (practically reflectionless) buffer zone that surrounds the domain of interest, where outgoing waves are forced to decay.

Obtaining the $\mathrm{P}$ - and S-wave velocity profiles of the soil in three-dimensional, arbitrarily heterogeneous domains, remains a challenging problem. The inverse medium problem at hand is notoriously ill-posed, computationally expensive, posed over a semi-infinite medium, and has challenging physics. Due to these complexities, and in order to render the problem tractable, current techniques rely on simplifying assumptions. We classify these simplifications as follows: a) limiting the spatial variability of the soil properties, whereby it is assumed that the soil is horizontally layered (one-dimensional) [25, 29, 33], or has properties varying only within a plane (two-dimensional) $[3,12,19,21]$; b) assuming that the

\footnotetext{
${ }^{1} \mathbf{x}=(x, y, z)$ is the position vector.

${ }^{2}$ The domain of interest is the region one aims to characterize.
} 


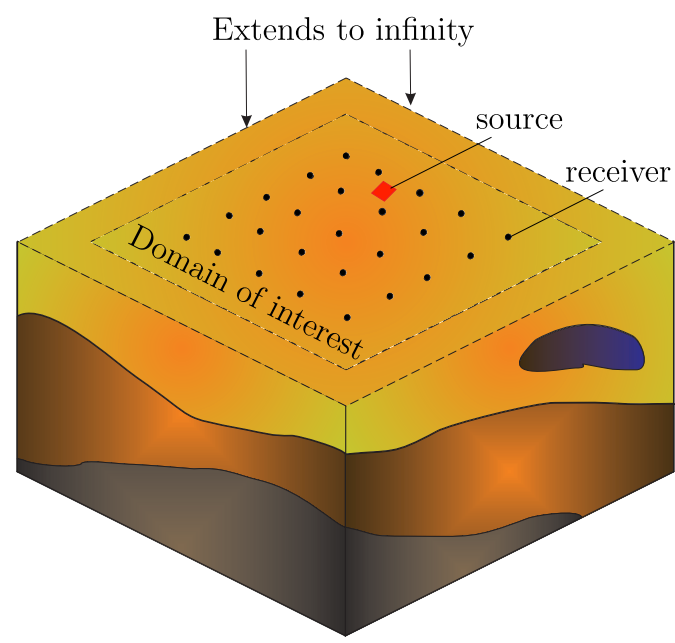

(a)

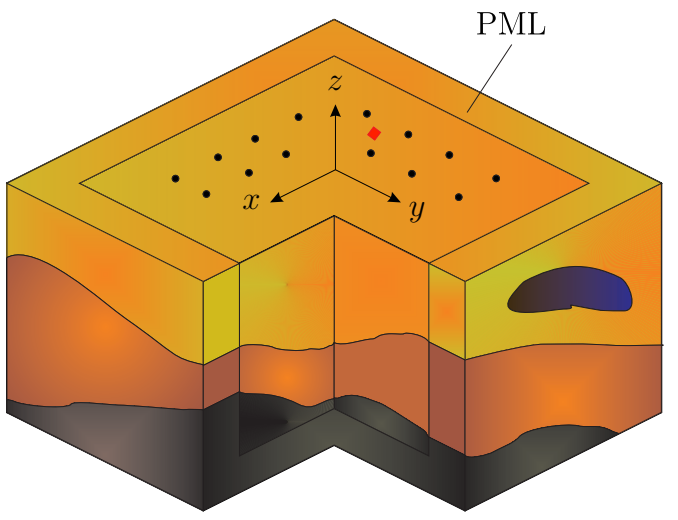

(b)

Figure 1: Problem definition: (a) interrogation of a heterogeneous semi-infinite domain by an active source; and (b) computational model truncated from the semi-infinite medium via the introduction of perfectlymatched-layers (PMLs).

measured response of the soil at sensor locations is due only to Rayleigh waves, thus neglecting other wave types, such as compressional and shear waves, as is the case in the SASW [33], or its close variant, the MASW method [30]; c) idealizing the soil medium, which is porous, and, generally, partially or fully saturated, as an elastic solid; d) imaging only one elastic property, such as the shear wave velocity or an equivalent counterpart $[2,11,27,28]$; and e) grossly simplifying the boundary conditions associated with the semi-infinite extent of the medium, due to the complexity and computational cost that a rigorous treatment would require $[11,36]$. In recent years, the ubiquity of parallel computers, and significant advances in computational geosciences, has created the opportunity of developing a toolkit that is capable of robust, accurate, and three-dimensional characterization of geotechnical sites.

In this paper, we extend recent advances in site characterization using full-waveform inversion (FWI) to the all-important case of three-dimensional site profiling using actual field data. In recent work [19], we described a methodology for accommodating field-data, which are inherently three-dimensional, into two-dimensional full-waveform-inversion-based software, whenever the site conditions would support plane strain assumptions. We demonstrated the method's viability by characterizing a site in Austin, Texas [19]. In this communication, we abandon the plane strain assumption, and consider arbitrary heterogeneity in three space dimensions. To be able to extend the framework described in [19] to the fully 
three-dimensional case, various algorithmic modifications are needed, including modifications to the forward three-dimensional wave simulator, per [15], to the inversion methodology, per [14], and to the manner the field experiment is designed and the associated field data are processed post-recording. We use the mathematical apparatus we described recently in $[13,14]$, and excerpt herein, but focus on the three-dimensional characterization of the NEES@UCSB site in Garner Valley, California, as a case study. To this end, we integrate recent advances in several areas. Specifically, we use: a) a parallel, three-dimensional, state-of-the-art wave simulation tool for domains terminated by PMLs [15]; b) a partialdifferential-equation (PDE)-constrained optimization framework, through which the misfit minimization between the measured response and a computed response is enforced [14]; c) regularization schemes to alleviate the ill-posedness and solution multiplicity inherent in inverse problems, where the regularization factor is computed adaptively at each inversion iteration $[14,20]$; d) continuation schemes that provide algorithmic robustness [20]; and e) a biasing scheme for the robust simultaneous inversion of both $c_{p}(\mathbf{x})$ and $c_{s}(\mathbf{x})$ profiles $[14,21]$.

Most inversion approaches result in profile reconstructions that are physically acceptable. Validation of the inverted profiles, in the absence of borehole data, becomes challenging, if not impossible. Herein, to aid in the validation of the inverted profiles, we use a set of control sensors. Specifically, out of the 35 sensors that we deployed at the NEES@UCSB site, we use only 30 of them to feed our full-waveform-inversion algorithms. The remaining 5 sensors serve as controls. We compute a time-history response corresponding to the full-waveform-inverted soil profiles, and compare them against the actual recorded field response at the location of the 5 control sensors. We report remarkable agreement at the control sensors.

The remainder of this paper is organized as follows: first, we review the simulation of elastic waves in a three-dimensional PML-truncated medium, followed by discussing a systematic mathematical framework for tackling the inverse medium problem. Next, we report on the design of the field experiment, data collection, data processing, and characterization of the NEES@UCSB site in Garner Valley, California, using full-waveform inversion. We then compare the resulting imaging against the profiling obtained from the SASW method. We also compute the time-history response of the site corresponding to the inverted profiles, and compare them against field measurements. Lastly, we conclude with summary remarks.

\section{Theoretical aspects - a review}

The mathematical development of our full-waveform-inversion-based methodology has been discussed in $[14,15]$. Here, for completeness, we excerpt key parts: there are two important components, namely, the forward wave simulator, and the inverse medium problem optimizer. 


\subsection{The forward problem}

We review first the numerical simulation of elastic wave propagation in a three-dimensional, semi-infinite, arbitrarily heterogeneous elastic medium, referred to as the forward problem. Due to the semi-infinite extent of the medium, domain truncation is necessary to arrive at a finite computational model. Limiting the unboundedness can be realized by placing PMLs at the truncation boundaries (Fig. 2(a)), such that, ideally, when outgoing waves travel through the reflectionless interface, they get attenuated within the PML buffer zone, as illustrated in Fig. 2(b).

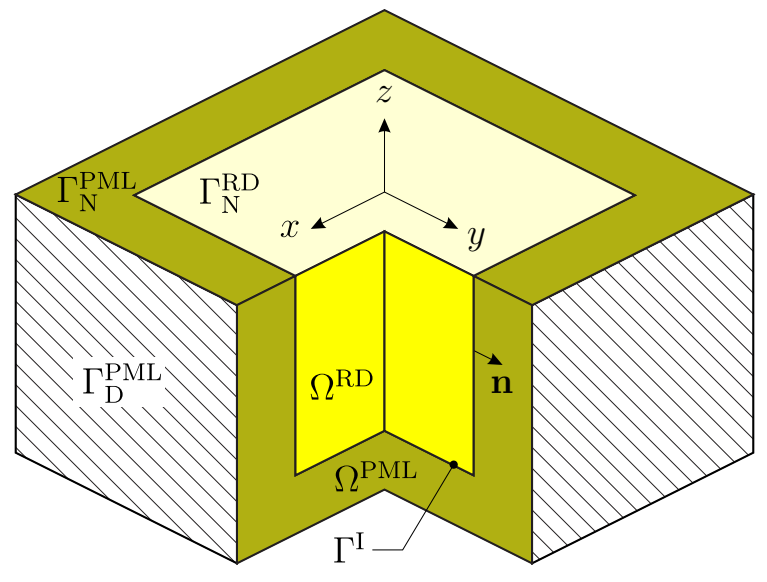

(a) PML-truncated semi-infinite domain

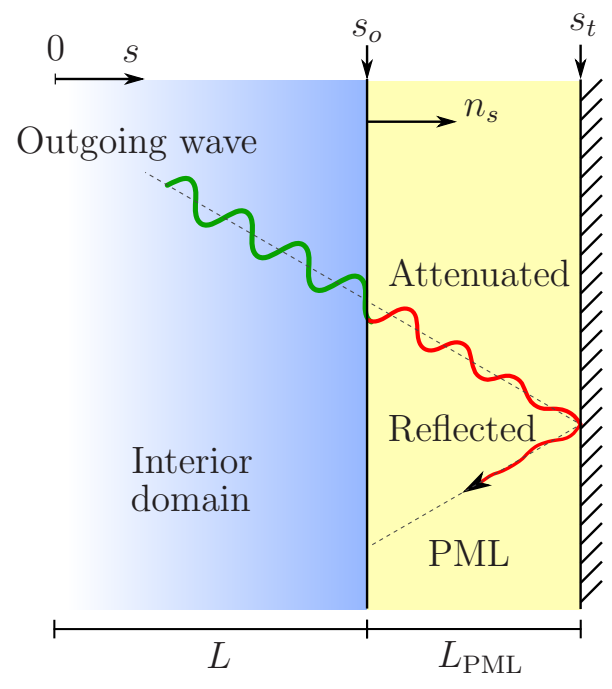

(b) A PML truncation boundary in the direction of coordinate $s$

Figure 2: Wave attenuation via perfectly-matched-layers (PMLs): (a) implementation at the computational domain level; (b) conceptual depiction of amplitude decay within the PML.

The forward problem needs to be solved repeatedly during the inversion process. Therefore, having a computationally efficient forward solver can influence the total computational cost significantly. To this end, we use a recently developed, state-of-the-art hybrid approach, which uses a displacement-stress formulation for the PML buffer, coupled with a standard displacement-only formulation for the interior (regular) domain. We refer to [15] and references therein for the complete development and parallel implementation of the forward wave solver. Herein, we only repeat the resulting coupled system of equations.

Accordingly, find $\mathbf{u}(\mathbf{x}, t)$ in $\Omega^{\mathrm{RD}} \cup \Omega^{\mathrm{PML}}$, and $\mathbf{S}(\mathbf{x}, t)$ in $\Omega^{\mathrm{PML}}$ (see Fig. 2(a) for domain and boundary designations), where $\mathbf{u}$ and $\mathbf{S}$ reside in appropriate function spaces and: 


$$
\begin{array}{lr}
\operatorname{div}\left\{\mu\left[\nabla \mathbf{u}+(\nabla \mathbf{u})^{T}\right]+\lambda(\operatorname{div} \mathbf{u}) \mathcal{I}\right\}=\rho \ddot{\mathbf{u}} & \text { in } \Omega^{\mathrm{RD}} \times \mathrm{J}, \\
\operatorname{div}\left(\dot{\mathbf{S}}^{T} \Lambda_{e}+\mathbf{S}^{T} \Lambda_{p}+\overline{\mathbf{S}}^{T} \Lambda_{w}\right)=\rho(a \ddot{\mathbf{u}}+b \dot{\mathbf{u}}+c \mathbf{u}+d \overline{\mathbf{u}}) & \text { in } \Omega^{\mathrm{PML}} \times \mathrm{J}, \\
a \ddot{\mathbf{S}}+b \dot{\mathbf{S}}+c \mathbf{S}+d \overline{\mathbf{S}}= & \\
\left.\mu\left[(\nabla \dot{\mathbf{u}}) \Lambda_{e}+\Lambda_{e}(\nabla \dot{\mathbf{u}})^{T}+(\nabla \mathbf{u}) \Lambda_{p}+\Lambda_{p}(\nabla \mathbf{u})^{T}+(\nabla \overline{\mathbf{u}}) \Lambda_{w}+\Lambda_{w}(\nabla \overline{\mathbf{u}})^{T}\right]+\operatorname{div}\left(\Lambda_{w} \overline{\mathbf{u}}\right)\right] \mathcal{I} & \text { in } \Omega^{\mathrm{PML}} \times \mathrm{J} . \\
\lambda\left[\operatorname{div}\left(\Lambda_{e} \dot{\mathbf{u}}\right)+\operatorname{div}\left(\Lambda_{p} \mathbf{u}\right)+\operatorname{lc}\right)
\end{array}
$$

The system is initially at rest, and subject to the following boundary and interface conditions:

$$
\begin{array}{ll}
\left\{\mu\left[\nabla \mathbf{u}+(\nabla \mathbf{u})^{T}\right]+\lambda(\operatorname{div} \mathbf{u}) \mathcal{I}\right\} \mathbf{n}=\boldsymbol{g}_{n} & \text { on } \Gamma_{\mathrm{N}}^{\mathrm{RD}} \times \mathrm{J}, \\
\left(\dot{\mathbf{S}}^{T} \Lambda_{e}+\mathbf{S}^{T} \Lambda_{p}+\overline{\mathbf{S}}^{T} \Lambda_{w}\right) \mathbf{n}=\mathbf{0} & \text { on } \Gamma_{\mathrm{N}}^{\mathrm{PML}} \times \mathrm{J}, \\
\mathbf{u}=\mathbf{0} & \text { on } \Gamma_{\mathrm{D}}^{\mathrm{PML}} \times \mathrm{J}, \\
\mathbf{u}^{\mathrm{RD}}=\mathbf{u}^{\mathrm{PML}} & \text { on } \Gamma^{\mathrm{I}} \times \mathrm{J}, \\
\left\{\mu\left[\nabla \mathbf{u}+(\nabla \mathbf{u})^{T}\right]+\lambda(\operatorname{div} \mathbf{u}) \mathcal{I}\right\} \mathbf{n}=\left(\dot{\mathbf{S}}^{T} \Lambda_{e}+\mathbf{S}^{T} \Lambda_{p}+\overline{\mathbf{S}}^{T} \Lambda_{w}\right) \mathbf{n} & \text { on } \Gamma^{\mathrm{I}} \times \mathrm{J},
\end{array}
$$

where temporal and spatial dependencies are suppressed for brevity; $\mathbf{u}$ is the displacement vector, $\rho$ is mass density of the medium, $\lambda$ and $\mu$ are the Lamé parameters, $\mathcal{I}$ is the secondorder identity tensor, $\dot{\mathbf{S}}$ represents the stress tensor, a dot $\left(^{*}\right)$ denotes differentiation with respect to time, and a bar $\left(^{-}\right)$indicates history of the subtended variable ${ }^{3} ; \Omega^{\mathrm{RD}}$ represents the interior (regular) domain, $\Omega^{\mathrm{PML}}$ denotes the region occupied by the PML buffer zone, $\Gamma^{\mathrm{I}}$ is the interface boundary between the interior and PML domains, $\Gamma_{\mathrm{N}}^{\mathrm{RD}}$ and $\Gamma_{\mathrm{N}}^{\mathrm{PML}}$ denote the free (top surface) boundary of the interior domain and PML, respectively, $\mathrm{J}=(0, T]$ is the time interval of interest, and $\boldsymbol{g}_{n}$ is the prescribed surface traction. Moreover, $\Lambda_{e}$, $\Lambda_{p}$, and $\Lambda_{w}$ are the so-called stretch tensors, which enforce dissipation of waves in $\Omega^{\mathrm{PML}}$, and $a, b, c$, and $d$ are products of certain elements of the stretch tensors [15]. Eq. (1a) is the governing PDE for the interior elastodynamic problem, whereas Eqs. (1b) and (1c) are the equilibrium and combined kinematic and constitutive equations, respectively, for the PML. Eqs. (2a) and (2b) describe the surface traction boundary condition for the interior domain and PML, respectively, and Eq. (2e) enforces the balance of tractions at the interface between the interior domain and PML. We remark that at the interface $\Gamma^{\mathrm{I}}$, $\Lambda_{e}=\mathcal{I}$, and $\Lambda_{p}=\Lambda_{w}=\mathbf{0}$.

\footnotetext{
${ }^{3}$ For instance, $\overline{\mathbf{u}}(\mathbf{x}, t)=\int_{0}^{t} \mathbf{u}(\mathbf{x}, \tau) d \tau$.
} 
We use a standard Galerkin finite element approach for the spatial discretization of the interior elastodynamic domain, where the unknowns are nodal displacements, whereas within the PML domain, displacements and stress components (i.e., $\mathbf{u}, \mathbf{S}$ ) are both treated as unknowns. It can be shown [15] that the following first-order system of ordinary differential equations results:

$$
\frac{d}{d t}\left[\begin{array}{c}
\mathbf{x}_{0} \\
\mathbf{x}_{1} \\
\mathbf{M} \mathbf{x}_{2}
\end{array}\right]=\left[\begin{array}{ccc}
\mathbf{0} & \mathbf{I} & \mathbf{0} \\
\mathbf{0} & \mathbf{0} & \mathbf{I} \\
-\mathbf{G} & -\mathbf{K} & -\mathbf{C}
\end{array}\right]\left[\begin{array}{c}
\mathbf{x}_{0} \\
\mathbf{x}_{1} \\
\mathbf{x}_{2}
\end{array}\right]+\left[\begin{array}{c}
\mathbf{0} \\
\mathbf{0} \\
\mathbf{f}^{\mathrm{st}}
\end{array}\right]
$$

where $\mathbf{M}, \mathbf{C}, \mathbf{K}, \mathbf{G}$, are system matrices, $\mathbf{x}_{0}=\overline{\mathbf{d}}^{\mathrm{st}}, \mathbf{x}_{1}=\mathbf{d}^{\mathrm{st}}, \mathbf{x}_{2}=\dot{\mathbf{d}}^{\mathrm{st}}, \mathbf{d}^{\mathrm{st}}=\left(\mathbf{u}_{h}^{T}, \mathbf{S}_{h}^{T}\right)^{T}$ is the vector of nodal unknowns comprising nodal displacements $\mathbf{u}_{h}$, in $\bar{\Omega}^{\mathrm{RD}} \cup \bar{\Omega}^{\mathrm{PML}}$, and nodal stress components $\mathbf{S}_{h}$, only in $\bar{\Omega}^{\mathrm{PML}}$, and $\mathbf{f}^{\text {st }}$ is the vector of applied forces. We use spectral elements with a Legendre-Gauss-Lobatto (LGL) quadrature rule to render $\mathbf{M}$ diagonal, and use an explicit fourth-order Runge-Kutta (RK-4) method for integrating (3) in time.

\subsection{The inverse medium problem}

Our goal is to find the spatial distribution of the P- and S-wave velocity profile of threedimensional, arbitrarily heterogeneous formations. Due to the expression of the material properties in terms of the Lamé parameters in Eqs. (1) and (2), the mathematical framework relies on inverting for $\lambda(\mathbf{x})$ and $\mu(\mathbf{x})$, followed by computing $c_{p}(\mathbf{x})$ and $c_{s}(\mathbf{x})$ through post-processing, according to:

$$
c_{s}(\mathbf{x})=\sqrt{\frac{\mu(\mathbf{x})}{\rho(\mathbf{x})}}, \quad c_{p}(\mathbf{x})=\sqrt{\frac{\lambda(\mathbf{x})+2 \mu(\mathbf{x})}{\rho(\mathbf{x})}} .
$$

The inverse medium problem can be formulated as the minimization of the misfit between the measured response $\mathbf{u}_{m}$, at sensor locations, and a computed response corresponding to a trial material profile:

$$
\min _{\lambda, \mu} \mathcal{J}(\lambda, \mu):=\frac{1}{2} \sum_{j=1}^{N_{r}} \int_{0}^{T} \int_{\Gamma_{m}}\left(\mathbf{u}-\mathbf{u}_{m}\right) \cdot\left(\mathbf{u}-\mathbf{u}_{m}\right) \delta\left(\mathbf{x}-\mathbf{x}_{j}\right) \mathrm{d} \Gamma d t+\mathcal{R}(\lambda, \mu)
$$

where $\mathbf{u}$ is the computed response, obtained from the forward problem, which is governed by the initial- and boundary-value problem (1), (2); $\mathcal{J}$ is the objective functional ${ }^{4}, N_{r}$ denotes the total number of sensors, $T$ is the total observation time, $\Gamma_{m}$ is the part of the ground surface where the sensor response has been recorded, $\delta\left(\mathbf{x}-\mathbf{x}_{j}\right)$ is the Dirac

\footnotetext{
${ }^{4}$ See $[4,7,9,10]$ for other possibilities.
} 
delta function, which formalizes measurements at sensor locations $\mathbf{x}_{j}$, and $\mathcal{R}(\lambda, \mu)$ is a regularization term, discussed next.

Inverse medium problems are notoriously ill-posed. They suffer from solution multiplicity; that is, material profiles that are very different from each other, and, potentially non-physical, can become solutions to the misfit minimization problem. Regularization of $\lambda$ and $\mu$ alleviates the ill-posedness. Herein, we use a Tikhonov regularization scheme [34], which penalizes large material gradients:

$$
\mathcal{R}(\lambda, \mu)=\frac{R_{\lambda}}{2} \int_{\Omega^{\mathrm{RD}}} \nabla \lambda \cdot \nabla \lambda \mathrm{d} \Omega+\frac{R_{\mu}}{2} \int_{\Omega^{\mathrm{RD}}} \nabla \mu \cdot \nabla \mu \mathrm{d} \Omega,
$$

where $R_{\lambda}$ and $R_{\mu}$ are the so-called $\lambda$ - and $\mu$-regularization factor, respectively, and control the amount of penalty imposed via (6) on the gradients of $\lambda$ and $\mu$.

A solution of (5) requires the satisfaction of the first-order optimality conditions [38]. We use the (formal) Lagrangian approach [31, 37] to impose the PDE-constraint (1), (2). Specifically, we introduce Lagrange multiplier vector function $\mathbf{w}$, and Lagrange multiplier tensor function $\mathbf{T}$, residing in appropriate function spaces, to enforce the initial- and boundary-value problem (1), (2) in its weak form. It can be shown [14] that the Lagrangian functional becomes:

$$
\begin{aligned}
\mathcal{L}(\mathbf{u}, \mathbf{S}, \mathbf{w}, \mathbf{T}, \lambda, \mu)=\frac{1}{2} \sum_{j=1}^{N_{r}} \int_{0}^{T} \int_{\Gamma_{m}}\left(\mathbf{u}-\mathbf{u}_{m}\right) \cdot\left(\mathbf{u}-\mathbf{u}_{m}\right) \delta\left(\mathbf{x}-\mathbf{x}_{j}\right) \mathrm{d} \Gamma d t+\mathcal{R}(\lambda, \mu) \\
\quad-\int_{0}^{T} \int_{\Omega^{\mathrm{RD}}} \nabla \mathbf{w}:\left\{\mu\left[\nabla \mathbf{u}+(\nabla \mathbf{u})^{T}\right]+\lambda(\operatorname{div} \mathbf{u}) \mathcal{I}\right\} \mathrm{d} \Omega d t \\
-\int_{0}^{T} \int_{\Omega^{\mathrm{PML}}} \nabla \mathbf{w}:\left(\dot{\mathbf{S}}^{T} \Lambda_{e}+\mathbf{S}^{T} \Lambda_{p}+\overline{\mathbf{S}}^{T} \Lambda_{w}\right) \mathrm{d} \Omega d t-\int_{0}^{T} \int_{\Omega^{\mathrm{RD}}} \mathbf{w} \cdot \rho \ddot{\mathbf{u}} \mathrm{d} \Omega d t \\
-\int_{0}^{T} \int_{\Omega^{\mathrm{PML}}} \mathbf{w} \cdot \rho(a \ddot{\mathbf{u}}+b \dot{\mathbf{u}}+c \mathbf{u}+d \overline{\mathbf{u}}) \mathrm{d} \Omega d t+\int_{0}^{T} \int_{\Gamma_{\mathrm{N}}^{\mathrm{RD}}} \mathbf{w} \cdot \boldsymbol{g}_{n} \mathrm{~d} \Gamma d t \\
-\int_{0}^{T} \int_{\Omega^{\mathrm{PML}}} \mathbf{T}:(a \ddot{\mathbf{S}}+b \dot{\mathbf{S}}+c \mathbf{S}+d \overline{\mathbf{S}}) \mathrm{d} \Omega d t \\
+\int_{0}^{T} \int_{\Omega^{\mathrm{PML}}} \mathbf{T}: \mu\left[(\nabla \dot{\mathbf{u}}) \Lambda_{e}+\Lambda_{e}(\nabla \dot{\mathbf{u}})^{T}+(\nabla \mathbf{u}) \Lambda_{p}+\Lambda_{p}(\nabla \mathbf{u})^{T}+(\nabla \overline{\mathbf{u}}) \Lambda_{w}+\Lambda_{w}(\nabla \overline{\mathbf{u}})^{T}\right] \\
+\mathbf{T}: \lambda\left[\operatorname{div}\left(\Lambda_{e} \dot{\mathbf{u}}\right)+\operatorname{div}\left(\Lambda_{p} \mathbf{u}\right)+\operatorname{div}\left(\Lambda_{w} \overline{\mathbf{u}}\right)\right] \mathcal{I} \mathrm{d} \Omega d t .
\end{aligned}
$$




\subsubsection{Optimality conditions}

We compute the first-order optimality conditions for (5) by using the Lagrangian functional (7). To this end, the Gâteaux derivative ${ }^{5}$ (or first variation) of the Lagrangian functional with respect to all variables must vanish.

We start by taking the derivatives of the Lagrangian functional $\mathcal{L}$ with respect to $\mathbf{w}$ and $\mathbf{T}$ in directions $\tilde{\mathbf{w}}$ and $\tilde{\mathbf{T}}$, residing in appropriate function spaces, and setting it to zero:

$$
\mathcal{L}^{\prime}(\mathbf{u}, \mathbf{S}, \mathbf{w}, \mathbf{T}, \lambda, \mu)(\tilde{\mathbf{w}}, \tilde{\mathbf{T}})=0 .
$$

This results in the forward problem, whose semi-discrete form is (3).

Next, we require that the derivative of $\mathcal{L}$ with respect to $\mathbf{u}$ and $\mathbf{S}$ in directions $\tilde{\mathbf{u}}$ and $\tilde{\mathbf{S}}$, residing in appropriate function spaces, vanish. This yields

$$
\mathcal{L}^{\prime}(\mathbf{u}, \mathbf{S}, \mathbf{w}, \mathbf{T}, \lambda, \mu)(\tilde{\mathbf{u}}, \tilde{\mathbf{S}})=0,
$$

which results in the adjoint problem, with semi-discrete form

$$
\frac{d}{d t}\left[\begin{array}{c}
\mathbf{y}_{0} \\
\mathbf{y}_{1} \\
\mathbf{M y}_{2}
\end{array}\right]=\left[\begin{array}{ccc}
\mathbf{0} & \mathbf{I} & \mathbf{0} \\
\mathbf{0} & \mathbf{0} & \mathbf{I} \\
\mathbf{G}^{T} & -\mathbf{K}^{T} & \mathbf{C}^{T}
\end{array}\right]\left[\begin{array}{l}
\mathbf{y}_{0} \\
\mathbf{y}_{1} \\
\mathbf{y}_{2}
\end{array}\right]+\left[\begin{array}{c}
\mathbf{0} \\
\mathbf{0} \\
\mathbf{f}^{\text {adj }}
\end{array}\right],
$$

where $\mathbf{y}_{0}=\overline{\mathbf{d}}^{\text {adj }}, \mathbf{y}_{1}=\mathbf{d}^{\text {adj }}, \mathbf{y}_{2}=\dot{\mathbf{d}}^{\text {adj }}, \mathbf{d}^{\text {adj }}=\left(\mathbf{w}_{h}^{T}, \mathbf{T}_{h}^{T}\right)^{T}$ is the vector of nodal unknowns comprising discrete values of $\mathbf{w}$ in $\bar{\Omega}^{\mathrm{RD}} \cup \bar{\Omega}^{\mathrm{PML}}$ and discrete values of $\mathbf{T}$ only in $\bar{\Omega}^{\mathrm{PML}}$, and $\mathbf{f}^{\text {adj }}$ is a vector comprising the misfit at sensor locations ${ }^{6}$. Moreover, system matrices $\mathbf{M}$, $\mathbf{C}, \mathbf{K}, \mathbf{G}$, are identical to those of the forward problem.

We remark that the adjoint problem (10) is a final-value problem (i.e., $\mathbf{y}_{0}(T)=\mathbf{0}$, $\mathbf{y}_{1}(T)=\mathbf{0}$, and $\mathbf{y}_{2}(T)=\mathbf{0}$ ), and thus, is solved backwards in time ${ }^{7}$. We use spectral elements to render $\mathbf{M}$ diagonal, and employ an explicit RK-4 scheme to integrate (10) in time [14].

Lastly, we require vanishing derivatives of $\mathcal{L}$ with respect to $\lambda$ and $\mu$ in directions $\tilde{\lambda}$ and $\tilde{\mu}$, which yield the gradients with respect to $\lambda$ and $\mu$, respectively:

$$
\begin{aligned}
& \mathcal{L}^{\prime}(\mathbf{u}, \mathbf{S}, \mathbf{w}, \mathbf{T}, \lambda, \mu)(\tilde{\lambda})=0, \\
& \mathcal{L}^{\prime}(\mathbf{u}, \mathbf{S}, \mathbf{w}, \mathbf{T}, \lambda, \mu)(\tilde{\mu})=0 .
\end{aligned}
$$

Discretization of (11) results in:

\footnotetext{
${ }^{5}$ See Appendix A for the definition and notation.

${ }^{6}$ Superscript "adj" refers to the adjoint problem.

${ }^{7}$ See $[22,35]$ for alternative approaches.
} 


$$
\begin{gathered}
\tilde{\mathbf{M}} \mathbf{g}^{\lambda}=R_{\lambda} \mathbf{g}_{\text {reg }}^{\lambda}+\mathbf{g}_{\text {mis }}^{\lambda}, \\
\tilde{\mathbf{M g}} \mathbf{g}^{\mu}=R_{\mu} \mathbf{g}_{\text {reg }}^{\mu}+\mathbf{g}_{\text {mis }}^{\mu},
\end{gathered}
$$

where $\tilde{\mathbf{M}}$ is a mass-like matrix, $\mathbf{g}^{\lambda}$ and $\mathbf{g}^{\mu}$ is the vector of discrete values of the gradient for $\lambda$ and $\mu$, respectively, and $\mathbf{g}_{\text {reg }}^{\lambda}, \mathbf{g}_{\text {reg }}^{\mu}$ and $\mathbf{g}_{\text {mis }}^{\lambda}, \mathbf{g}_{\text {mis }}^{\mu}$ are the associated vectors corresponding to the regularization-part and misfit-part of $\mathbf{g}^{\lambda}$ and $\mathbf{g}^{\mu}$. We refer to [14] for matrix and vector definitions, guidelines for selecting the regularization factors $R_{\lambda}, R_{\mu}$, and a comprehensive treatment of the inverse medium problem in three-dimensional PML-truncated domains.

\subsubsection{Gradient-based optimization}

In a gradient-based scheme, discrete material properties are updated iteratively, using the gradient of the objective functional (5) with respect to $\lambda$ and $\mu$, until the misfit is minimized. We start with an assumed initial spatial distribution of the material parameters $\lambda(\mathbf{x})$ and $\mu(\mathbf{x})$, and solve the state problem (3) to obtain $\mathbf{d}^{\text {st }}=\left(\mathbf{u}_{h}^{T}, \mathbf{S}_{h}^{T}\right)^{T}$. With the misfit known, we solve the adjoint problem (10) and obtain $\mathbf{d}^{\text {adj }}=\left(\mathbf{w}_{h}^{T}, \mathbf{T}_{h}^{T}\right)^{T}$. With $\mathbf{u}_{h}$ and $\mathbf{w}_{h}$ known, the (reduced) material gradients, i.e., $\mathbf{g}^{\lambda}$ and $\mathbf{g}^{\mu}$, can be computed from (12). Next, the vector of material values, at iteration $k+1$, can be obtained by using a search direction via:

$$
\begin{aligned}
& \boldsymbol{\lambda}_{k+1}=\boldsymbol{\lambda}_{k}+\alpha_{k}^{\lambda} \boldsymbol{s}_{k}^{\lambda}, \\
& \boldsymbol{\mu}_{k+1}=\boldsymbol{\mu}_{k}+\alpha_{k}^{\mu} \boldsymbol{s}_{k}^{\mu},
\end{aligned}
$$

where $\boldsymbol{\lambda}$ and $\boldsymbol{\mu}$ comprise the vector of discrete values for $\lambda$ and $\mu$, respectively, $\alpha_{k}^{\lambda}, \alpha_{k}^{\mu}$ are step lengths, and $\boldsymbol{s}_{k}^{\lambda}, \boldsymbol{s}_{k}^{\mu}$ are the search directions for $\boldsymbol{\lambda}_{k}$ and $\boldsymbol{\mu}_{k}$. Herein, we use the L-BFGS method to compute the search directions $[26]^{8}$. Moreover, to ensure sufficient decrease of the objective functional at each inversion iteration, we employ an Armijo backtracking line search [26]. Furthermore, to alleviate the ill-posedness, and assist the inversion process, we bias the search direction of $\lambda$ based on that of $\mu$ at the early iterations of the inversion process, as detailed in [14].

\section{Field experiments}

We report next on the design and data processing of two field experiments that we performed at the NEES@UCSB site in Garner Valley, California. The first field experiment is aimed at collecting data for the three-dimensional $c_{p}$ and $c_{s}$ profiling of the site, using the full-waveform inversion methodology discussed earlier, and the second experiment seeks

\footnotetext{
${ }^{8}$ We store $m=15$ L-BFGS vectors.
} 
to collect data for reconstructing a one-dimensional $c_{s}$ profile, using the SASW method. We first introduce the equipment that we used in the field experiments, followed by the experimental layout. Next, we discuss the key characteristics of the surface load signal, and comment on the post-processing needs of the collected data. We then present the full-waveform-inversion-based $c_{p}$ and $c_{s}$ profiles of the site, and compare them against the $c_{s}$ profiling obtained from the SASW method. Lastly, we show simulated time-history response at control sensor locations due to the soil profiles obtained from the full-waveform inversion, and that of the SASW method. We compare the simulated time histories with actual field measurements in an attempt to assess the quality of the inversion.

\subsection{Field equipment}

We use the T-Rex Vibroseis of the NEES@UTexas equipment site for applying loads on the ground surface (Fig. 3(a)). The equipment can apply vertical loads with a maximum force amplitude of $267 \mathrm{kN}$ within a frequency range of 12 to $120 \mathrm{~Hz}$. The Vibroseis is also capable of applying loads outside this frequency range, albeit, at a lower amplitude. We record the resulting motion on the ground surface with 1-Hz geophones (Fig. 3(b)).

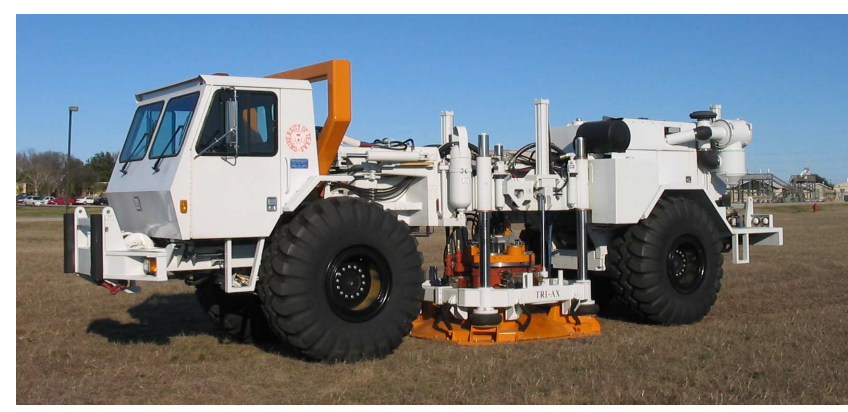

(a) NEES@UTexas T-Rex Vibroseis: the largest triaxial seismic vibration truck in the world

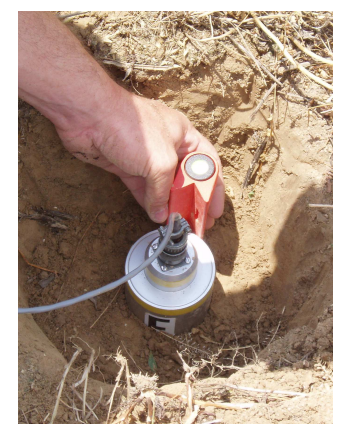

(b) NEES@UTexas 1-Hz geophone

Figure 3: Equipment used for the field experiments at the NEES@UCSB site.

\subsection{Experimental site and layout}

We aim at the seismic site characterization of a subregion of the NEES@UCSB site in Southern California. It is located in a narrow valley, within the Peninsular Ranges batholith, $23 \mathrm{~km}$ east of Hemet, $20 \mathrm{~km}$ southwest of Palm Springs, and is situated just $7 \mathrm{~km}$ from the San Jacinto fault, and $35 \mathrm{~km}$ from the San Andreas fault [1].

We consider a portion of the site of length and width $66 \mathrm{~m} \times 68 \mathrm{~m}$, and $40 \mathrm{~m}$ depth. The experiment layout is shown in Fig. 4. The main grid lines are $10 \mathrm{~m}$ apart. The sensors are shown with (black) solid circles, while sources are indicated by (red) squares. Overall, 
we "shake" the site at 12 locations, and record the site's response using 35 sensors. The experiment was performed on March 13, 2012 [6].

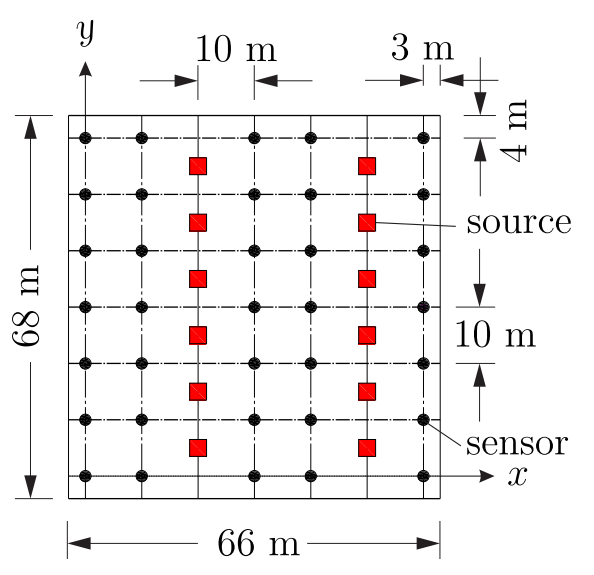

Figure 4: Field experimental layout

\subsection{Surface load signal characteristics}

Loads that have a broad range of frequencies can probe the site more effectively than those containing only a few frequencies. In this study, we use chirps, since their frequency content can be adjusted to increase progressively from low to higher frequencies with respect to time. They have been used successfully in site characterization [19], and are also common in radar and other geophysical applications. Specifically, we use a linear chirp of the form:

$$
f(t)=\sin \left(2 \pi\left(f_{0}+\frac{k t}{2}\right) t\right)
$$

where $f_{0}$ is the starting frequency, typically decided based on the geophone's resonant frequency, and $k$ is the chirp rate, which controls how fast the frequency content of the signal increases with respect to time. We use characteristic parameters $f_{0}=3 \mathrm{~Hz}$, and $k=2.8 \mathrm{~s}^{-2}$, with a total active time duration of $2.5 \mathrm{~s}$, which results in a loading signal with strong frequency components within the range of $3-10 \mathrm{~Hz}$. Figure 5 shows the (theoretical) time history of the load and its corresponding Fourier spectrum.

It is worth mentioning that for the numerical solution of the adjoint problem (10), and evaluation of the gradients (12), solution history of the state variables ${ }^{9}$ is required at all time steps. Thus, a signal with a longer time duration may necessitate more storage ${ }^{10}$,

\footnotetext{
${ }^{9}$ Storage of the adjoint variables can be avoided by updating the gradients on the fly, while solving the adjoint problem backwards in time.

${ }^{10}$ Checkpointing strategies may be exploited to relieve storage requirements, albeit at additional computational cost [11].
} 

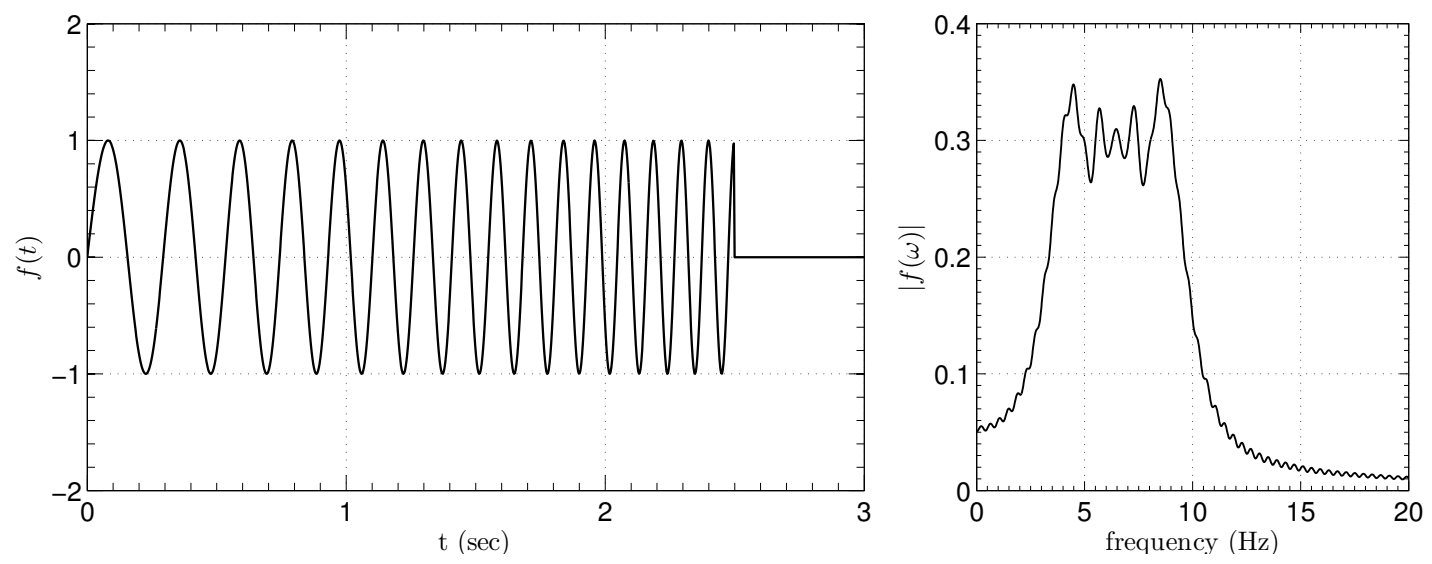

Figure 5: Theoretical time history of the chirp load and its Fourier spectrum.

in addition to increasing the computational cost. Therefore, the temporal duration of the signal should be only long enough to probe the site effectively.

We remark that T-Rex is unable to generate a chirp signal in its exact form, nor is this vital. Thus, by installing accelerometers on its baseplate, we compute the force that the equipment applies on the ground surface. This load is then used in the solution of the forward problem (3).

\subsection{Processing of the collected field data}

The recorded field data is inevitably contaminated with noise. We identify the parts of the signal (in the frequency-domain) with low signal-to-noise ratio, and eliminate them, by using an equiripple finite-impulse-response (FIR) filter, which preserves the signal's phase information [32]. Specifically, we use a bandpass filter, with high and low cuts of $1.5 \mathrm{~Hz}$ and $14 \mathrm{~Hz}$, respectively, and high and low slopes of $66.6 \mathrm{~dB} / \mathrm{Hz}$ and $15 \mathrm{db} / \mathrm{Hz}$, respectively. We use a sampling frequency of $200 \mathrm{~Hz}$ for digital data collection, which, according to the Nyquist sampling theorem [32], is adequate, and prevents aliasing, if the recorded data is contaminated with noise up to $100 \mathrm{~Hz}$. Moreover, to reduce the effects of ambient noise, we repeat each loading five times, and use the averaged recording for inversion.

Next, we present a subset of the processed recorded motion at select sensor locations. Specifically, we consider the displacement time-history at four sensors, placed at $(x, y)=$ $(0,60),(40,60),(0,10),(40,10) \mathrm{m}$, due to loading at $(x, y)=(20,35) \mathrm{m}$. These sensors are equidistant from the source, and are shown with solid circles in Fig. 6, while the source is indicated by a square. The load and displacement time-histories at these locations are shown in Figs. 7 and 8, respectively. Figure 8 depicts both the raw sensor recordings, as well as the processed records, per the earlier outlined procedure. Notice that, although 
there is general agreement between the time-histories of the equidistant sensors, there are differences, which are indicative of the heterogeneity of the site.

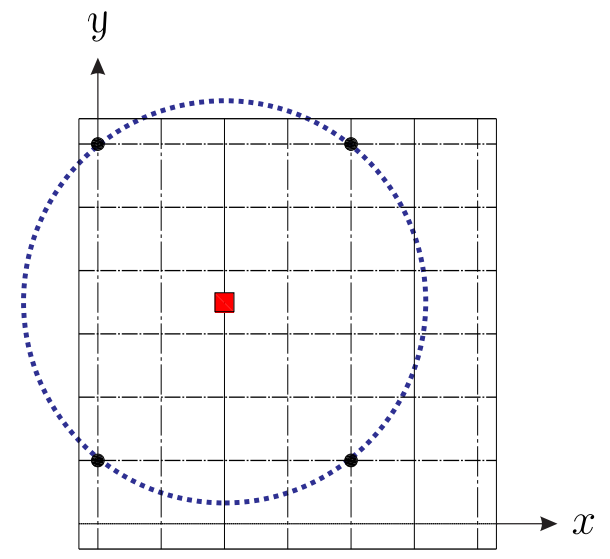

Figure 6: A source located at $(x, y)=(20,35) \mathrm{m}$, and four equidistant sensors.

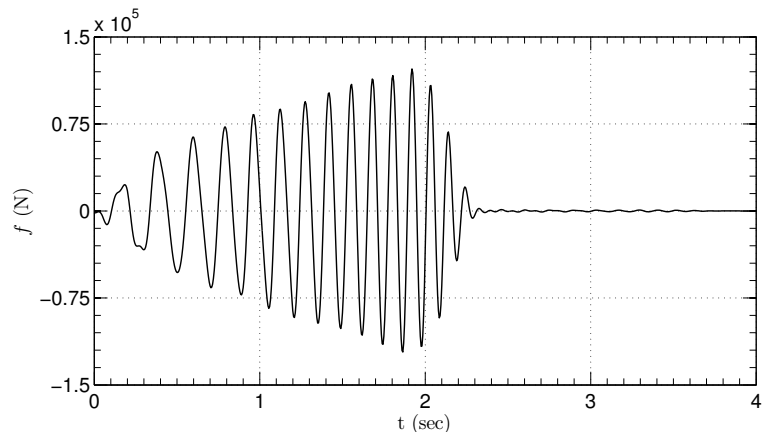

Figure 7: Time-history of the source load shown in Fig. 6.

\subsection{Full-waveform inversion using field data}

Based on the inversion framework discussed in the preceding sections, we use the collected field data to compute the three-dimensional $c_{p}$ and $c_{s}$ profiles of the probed site. From the 35 sensors where the time-history response of the site was recorded, we use only 30 of them to drive the inversion, and use the remaining 5 control sensors to validate the resulting profiles. The two sets of sensors are shown in Fig. 9.

We consider a cubic (regular) domain of length, width, and depth $66 \mathrm{~m} \times 68 \mathrm{~m} \times 40 \mathrm{~m}$. A $10 \mathrm{~m}$-thick PML is placed at the truncation boundaries. For the PML parameters, we choose $\alpha_{o}=5, \beta_{o}=500 \mathrm{~s}^{-1}$, and a quadratic profile for the attenuation functions, i.e., $m=2$ (see [15] for notation and other details regarding the PML parameters). The 


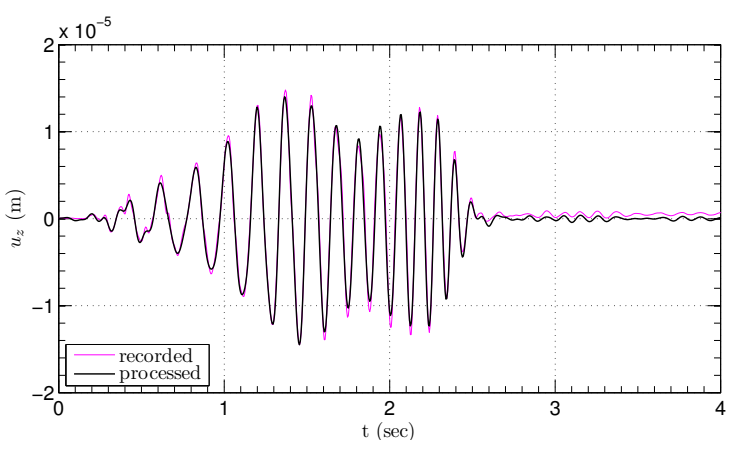

(a) $(x, y)=(0,60) \mathrm{m}$

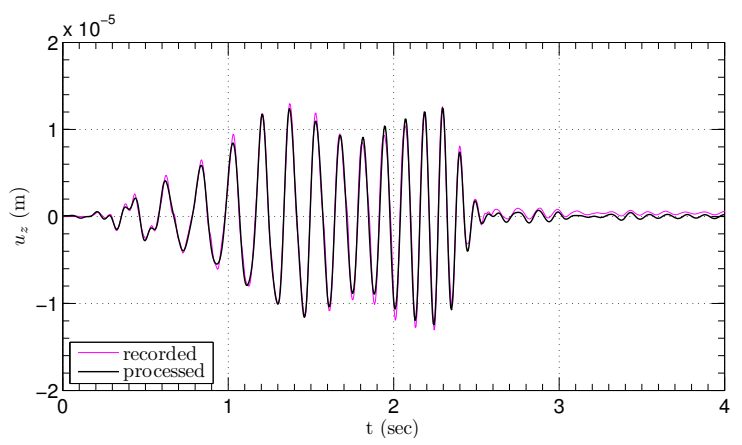

(c) $(x, y)=(0,10) \mathrm{m}$

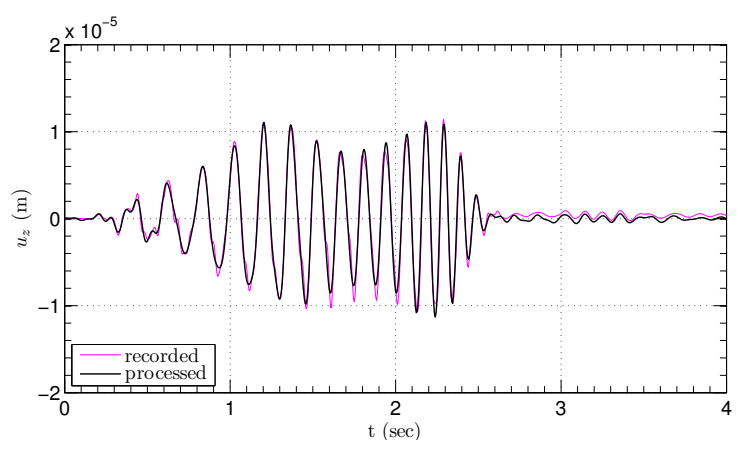

(b) $(x, y)=(40,60) \mathrm{m}$

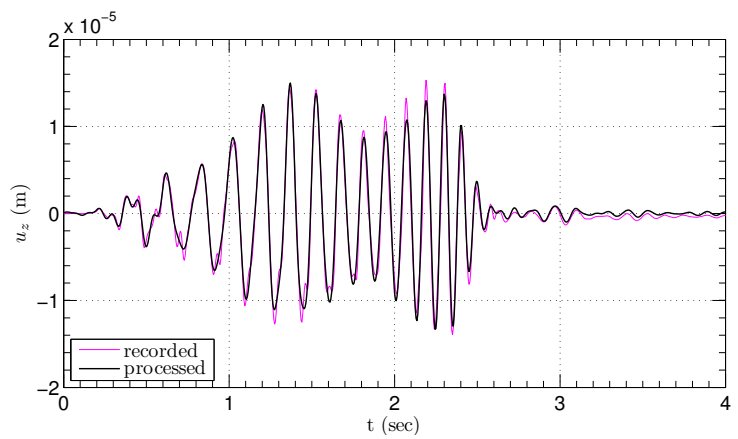

(d) $(x, y)=(40,10) \mathrm{m}$

Figure 8: Vertical displacement time-histories at the four sensors shown in Fig. 6. 

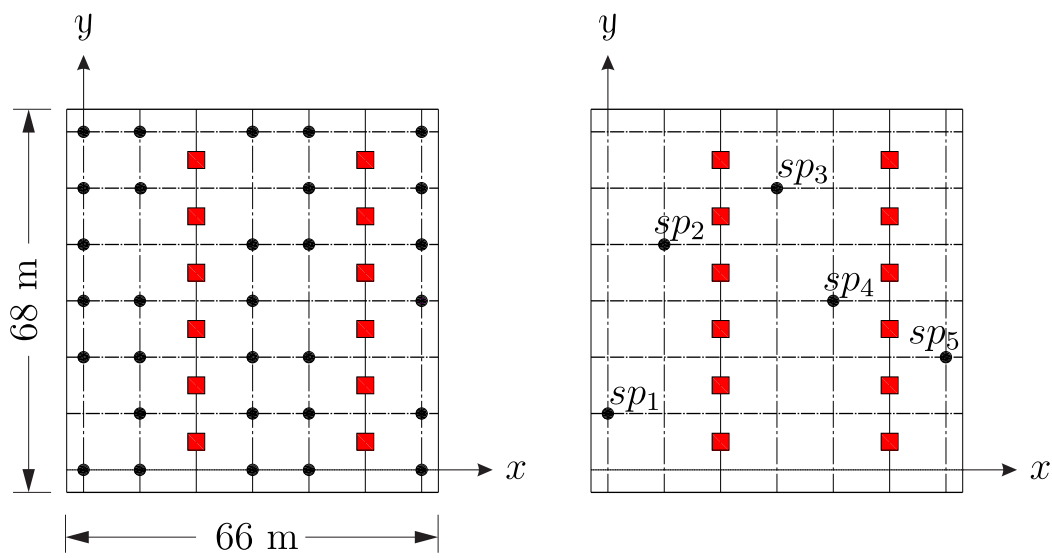

Figure 9: Sensors used for full-waveform inversion (left), and validation (right), respectively. Control sensors are marked $s p_{1}$ through $s p_{5}$.

mass density is considered to be $\rho=1760 \mathrm{~kg} / \mathrm{m}^{3}$ for $-2 \mathrm{~m} \leq z \leq 0, \rho=1880 \mathrm{~kg} / \mathrm{m}^{3}$ for $-4 \mathrm{~m} \leq z \leq-2 \mathrm{~m}$, and $\rho=2000 \mathrm{~kg} / \mathrm{m}^{3}$ for $-40 \mathrm{~m} \leq z \leq-4 \mathrm{~m}$, according to prior site investigations ${ }^{11}$. The material properties at the interfaces $\Gamma^{\mathrm{I}}$ are extended into the PML. The interior and PML domains are discretized by quadratic hexahedral spectral elements with $2 \mathrm{~m}$ sides (i.e., 27-noded bricks, and quadratic-quadratic pairs of approximation for displacement and stress components in the PML, and, also, quadratic approximation for material properties). The time step is $\Delta t=10^{-3} \mathrm{~s}$. This discretization leads to 2,433, 408 state unknowns, and 379,086 material parameters. To probe the site, we use the chirp signal discussed in Section 3.3. Owing to linearity, we apply all of the 12 loads simultaneously, and consider their superimposed responses at each sensor location, after synchronizing the recorded response.

Due to the construction of the chirps, their frequency content increases with time, which can be exploited to advantage by further regularizing the inversion process. That is, we start with a portion of the signal to drive inversion, and as the inversion evolves, we increase the temporal duration of the signal, thus, progressively including higher frequencies, which allows for profile refinement $[19,23,24]$. Table 1 shows the details of this process.

The inversion process begins with an initial guess for the material profiles, which is then updated iteratively, until the objective functional (5) is minimized. The speed of convergence, and even the success of the inversion itself, relies heavily on the initial assumption for the material profiles. Although, in principle, it is possible to start with a homogeneous initial guess ${ }^{12}$, oftentimes, prior information about the site may be of assistance to

\footnotetext{
${ }^{11}$ We do not invert for the mass density.

${ }^{12} \mathrm{~A}$ homogeneous initial guess may necessitate a multi-scale approach, using a sequence of finer grids, to avoid trapping in local minima $[8,16]$.
} 
Table 1: Parameters used during each inversion stage.

\begin{tabular}{lrrr}
\hline Stage & $\begin{array}{r}\text { temporal } \\
\text { duration }\end{array}$ & $\begin{array}{r}\text { normalized regularization } \\
\text { factor parameter } \varrho[14]\end{array}$ & $\begin{array}{r}\text { cumulative } \\
\text { iterations }\end{array}$ \\
\hline 1 & 0.5 & 0.60 & 10 \\
2 & 1.0 & 0.55 & 180 \\
3 & 1.5 & 0.50 & 350 \\
4 & 2.0 & 0.45 & 430 \\
5 & 2.5 & 0.40 & 460 \\
6 & 3.0 & 0.35 & 500 \\
\hline
\end{tabular}

the optimizer. For instance, a smoothed profile obtained from the SASW method may be used as the initial guess: specifically, we performed SASW at one location, smoothed out the staircase, layered profile the SASW produced, and used it as initial guess for the three-dimensional full-waveform inversion.

After 500 iterations, the misfit between the measured response and the computed response becomes reasonably small, with no strong update for the material parameters. The corresponding three-dimensional $c_{p}$ and $c_{s}$ profiles for the Garner Valley site are shown in Fig. 10 and 11, respectively, whereas Figs. 12 and 13 show the cross sections of the velocity profiles at $x=0,20,40$, and $60 \mathrm{~m}$. These profiles indicate that the site under investigation is heterogeneous and layered. Having obtained the $c_{p}(\boldsymbol{x})$ and $c_{s}(\boldsymbol{x})$ profiles, we then seek to compute the Poisson's ratio $\nu(\boldsymbol{x})$ everywhere in the probed region ${ }^{13}$. The Poisson's ratio distribution is shown in Fig. 14. Except for a small region, concentrated around $x=60 \mathrm{~m}$, the Poisson's ratio varies between 0.30 to 0.35 , which is quite reasonable. Since the Pand S-wave velocities were obtained by simultaneously inverting for both velocities, and since we had not imposed a constraint on Poisson's ratio, the fact that the Poisson's ratio ended up being within a narrow and physically acceptable range is a strong indicator of the correctness of the inverted profiles.

\subsection{Comparison with $S A S W$}

Having obtained the $c_{p}$ and $c_{s}$ profiles of the site via full-waveform inversion, next, we compare them against the $c_{s}$ profile obtained from the SASW method. While full-waveform inversion uses the complete waveform of the recorded response at sensor locations, and is capable of imaging three-dimensional, arbitrarily heterogeneous formations, SASW considers only surface (Raleigh) waves, and outputs a one-dimensional layered profile for the shear wave velocity: the method relies on the dispersive nature of Raleigh waves in layered media. A material profile, based on the SASW method, is obtained by: a) constructing an experimental dispersion curve, based on a field experiment; b) constructing a theoretical

\footnotetext{
${ }^{13}$ The computation is based on post-processing the inverted-for distributions of the Lamé parameters $\lambda(\boldsymbol{x})$ and $\mu(\boldsymbol{x})$, according to $\nu(\boldsymbol{x})=\lambda(\boldsymbol{x}) / 2(\lambda(\boldsymbol{x})+\mu(\boldsymbol{x}))$.
} 


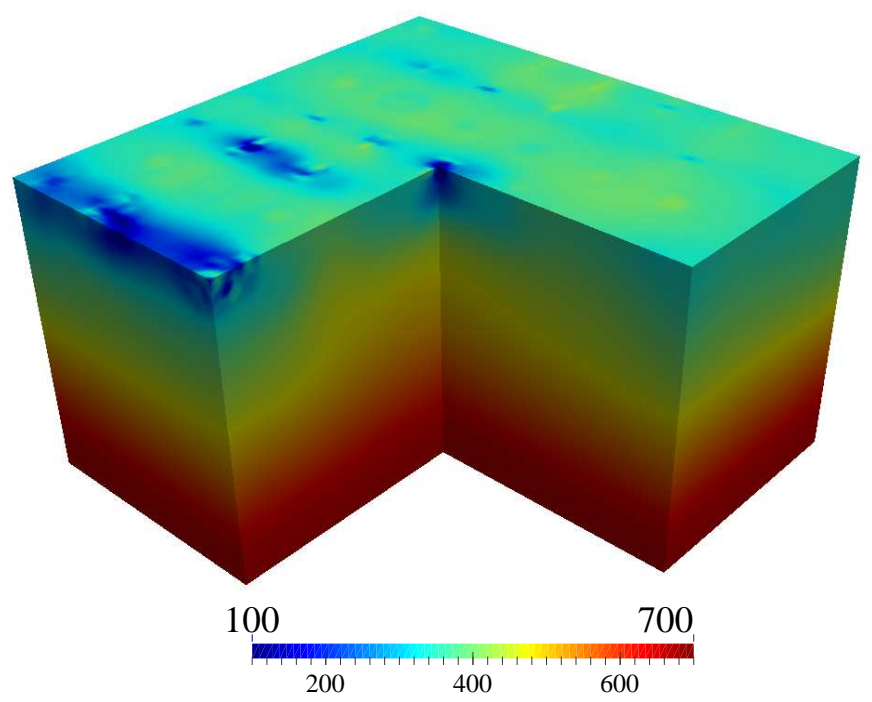

Figure 10: P-wave velocity (in $\mathrm{m} / \mathrm{s}$ ) profile of the site obtained via full-waveform inversion. The vertical section cuts through the domain from $(x, y)=(30,60)$ (right), to $(30,30)$, to $(60,30)$ (left).

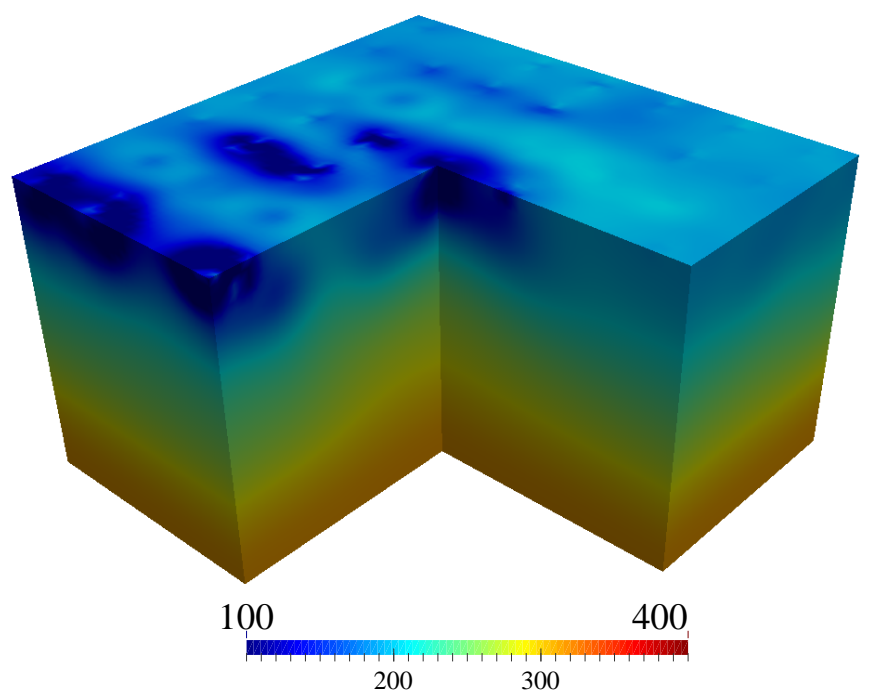

Figure 11: S-wave velocity (in $\mathrm{m} / \mathrm{s}$ ) profile of the site obtained via full-waveform inversion. The vertical section cuts through the domain from $(x, y)=(30,60)$ (right), to $(30,30)$, to $(60,30)$ (left). 

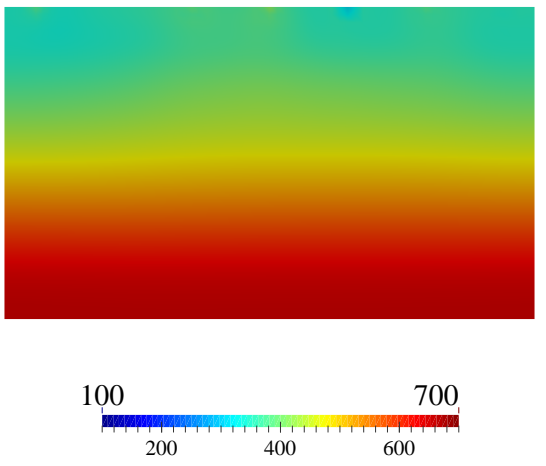

(a) $c_{p}(m / s)$ at $x=0 \mathrm{~m}$
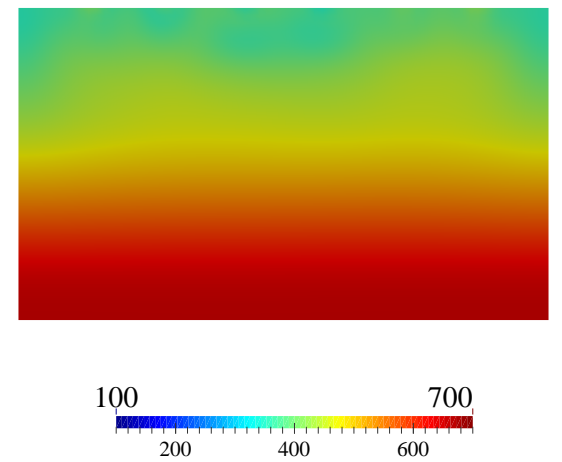

(c) $c_{p}(\mathrm{~m} / \mathrm{s})$ at $x=20 \mathrm{~m}$
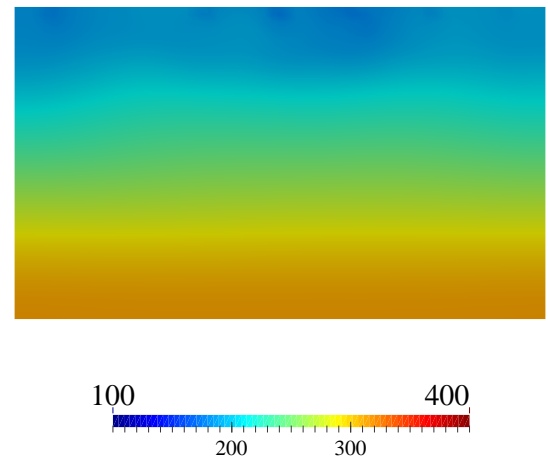

(b) $c_{s}(\mathrm{~m} / \mathrm{s})$ at $x=0 \mathrm{~m}$
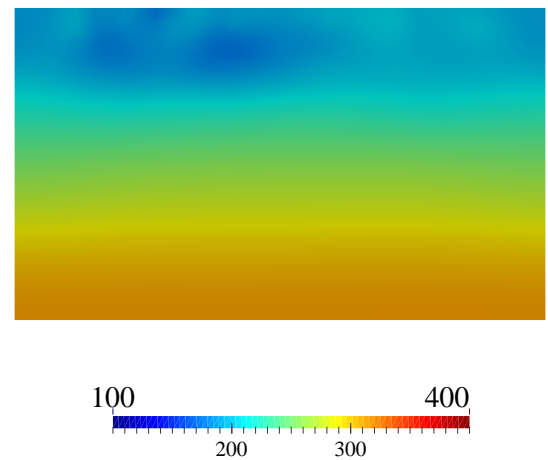

(d) $c_{s}(\mathrm{~m} / \mathrm{s})$ at $x=20 \mathrm{~m}$

Figure 12: Cross sections of $c_{p}$ and $c_{s}$ profiles obtained via full-waveform inversion at $x=0 \mathrm{~m}$ and $x=20 \mathrm{~m}$. 

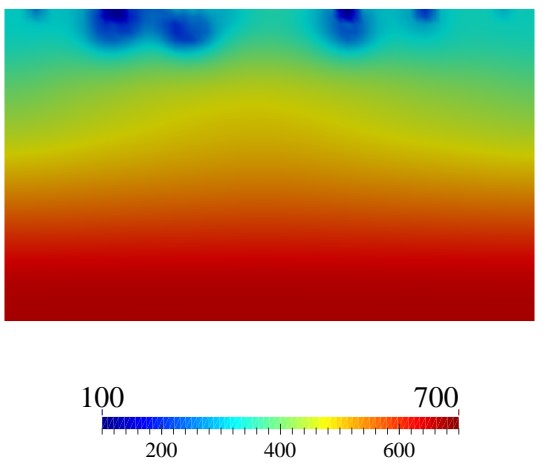

(a) $c_{p}(m / s)$ at $x=40 \mathrm{~m}$
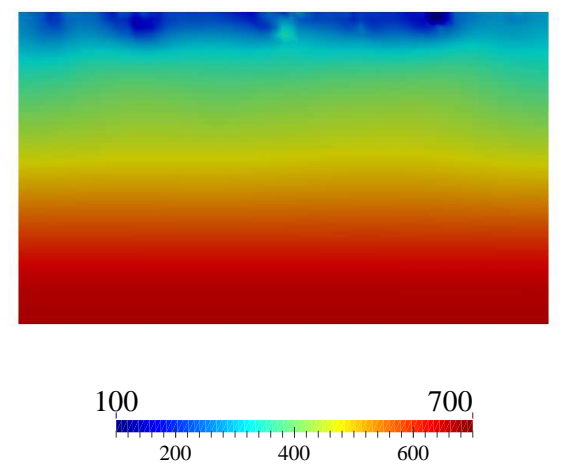

(c) $c_{p}(\mathrm{~m} / \mathrm{s})$ at $x=60 \mathrm{~m}$
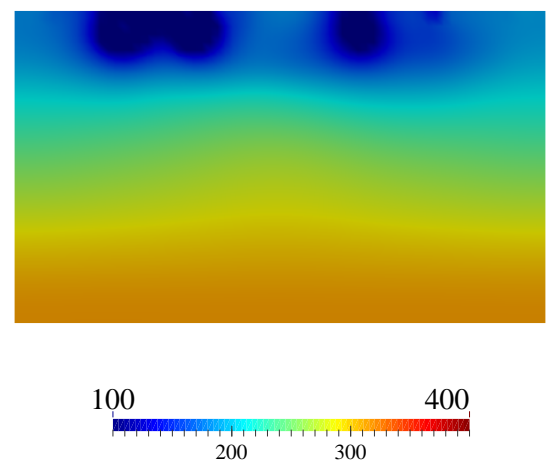

(b) $c_{s}(\mathrm{~m} / \mathrm{s})$ at $x=40 \mathrm{~m}$
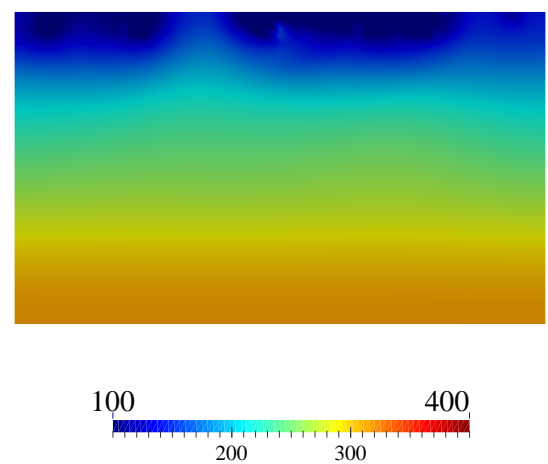

(d) $c_{s}(\mathrm{~m} / \mathrm{s})$ at $x=60 \mathrm{~m}$

Figure 13: Cross sections of $c_{p}$ and $c_{s}$ obtained via full-waveform inversion at $x=40 \mathrm{~m}$ and $x=60 \mathrm{~m}$. 


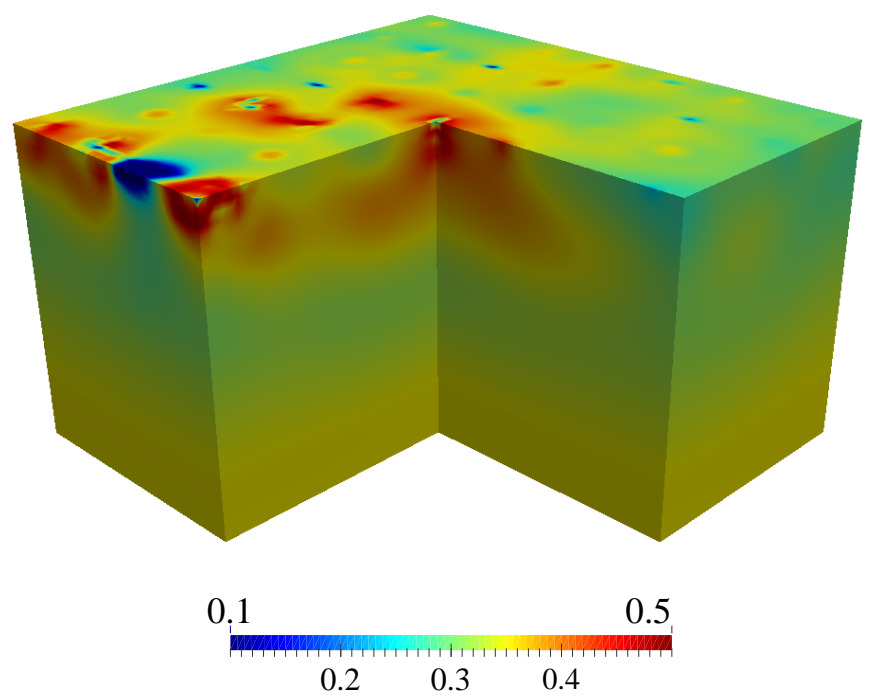

Figure 14: Poisson's ratio obtained via processing of the full-waveform inversion profiles.

dispersion curve, by assuming a trial distribution for the material properties of a layered profile; and c) varying the material properties of the layered profile, and repeating the previous step, until a match between the experimental dispersion curve, and the theoretical dispersion curve is attained $[18,33]$.

We performed field tests using the SASW method to obtain the $c_{s}$ profile of the site along two array lines. The profiles are obtained along $x=0 \mathrm{~m}$ and $40 \mathrm{~m}$, which are marked with red lines in Fig. 15, and are shown in Fig. 16. The corresponding $c_{s}$ profiles obtained from the full-waveform inversion at three different locations along these lines are also shown in these plots. There is very good agreement between the profiles at near-surface regions (top $25 \mathrm{~m}$ ), while at the deeper part of the site, the full-waveform-inversion-based $c_{s}$-profile predicts lower values than those obtained via SASW.

\subsection{Time-history comparisons at sensor locations}

We compute the time-history response corresponding to a forward wave simulation of the site, based on profiles obtained from full-waveform inversion and that of the SASW method $^{14}$ (Fig. 17), and compare them against the measured field response at various sensor

\footnotetext{
${ }^{14}$ To compute the $c_{p}$ profile for the SASW method we set $\nu=0.33$ when $z>-6 \mathrm{~m}$, and set $c_{p}=1500 \mathrm{~m} / \mathrm{s}$ otherwise. The $z=-6 \mathrm{~m}$ corresponds to an estimation of the location of the water table. Furthermore, since the SASW profiles for the two array lines are almost identical, we average the velocity values for time-history simulations.
} 


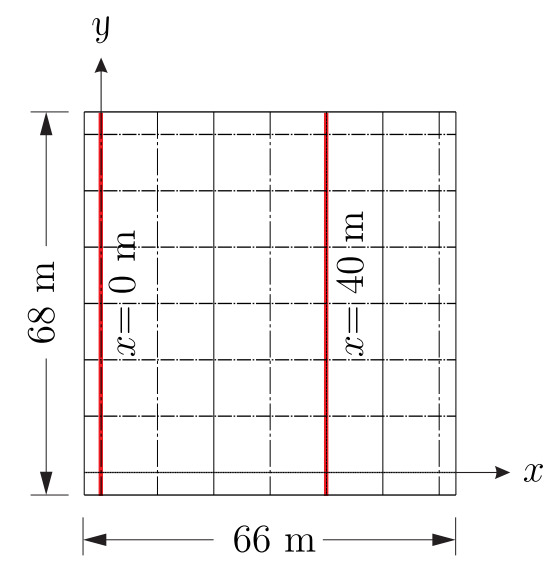

Figure 15: Locations of the SASW-method array lines

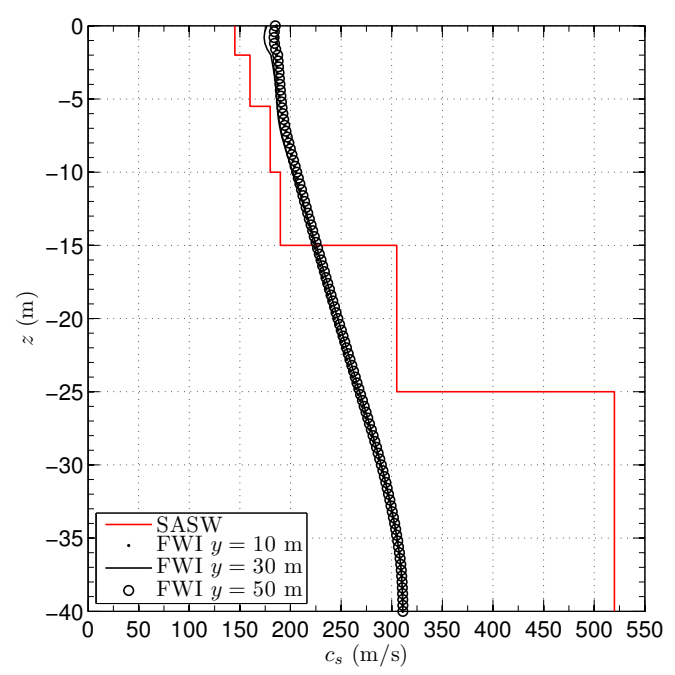

(a) $x=0 \mathrm{~m}$

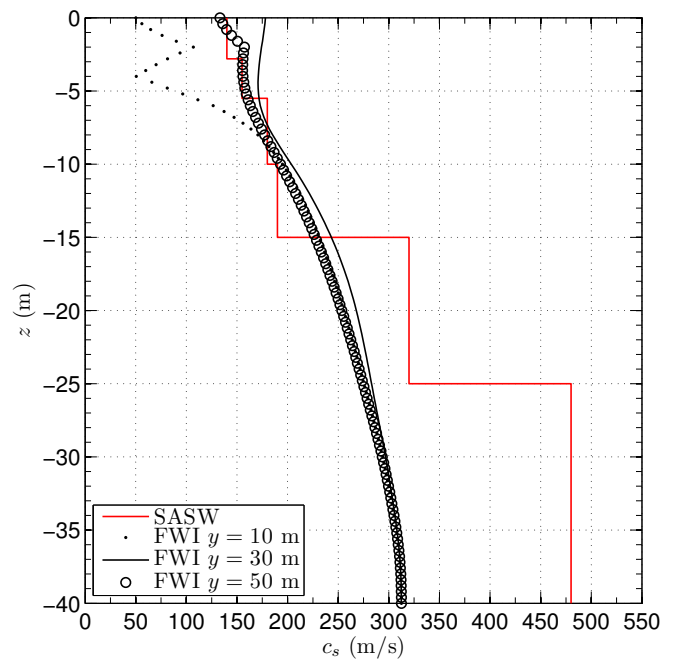

(b) $x=40 \mathrm{~m}$

Figure 16: Comparison of site shear wave velocity profiles obtained via SASW and full-waveform inversion (FWI); SASW profile is horizontally layered; FWI is spatially variable: profiles are shown along 3 array lines at $y=10 \mathrm{~m}, 30 \mathrm{~m}, 50 \mathrm{~m}$ 
locations. We perform the comparison in two parts: 1) for the 5 control sensors $s p_{1}-s p_{5}$, which were not used during the inversion, shown in Fig. 9; and 2) for the remaining 30 sensors that were used during the inversion process. Part 1 is an attempt to validate the profiles obtained via the full-waveform-inversion-based methodology, while part 2 gauges the success of the misfit-minimization algorithm when using field data.

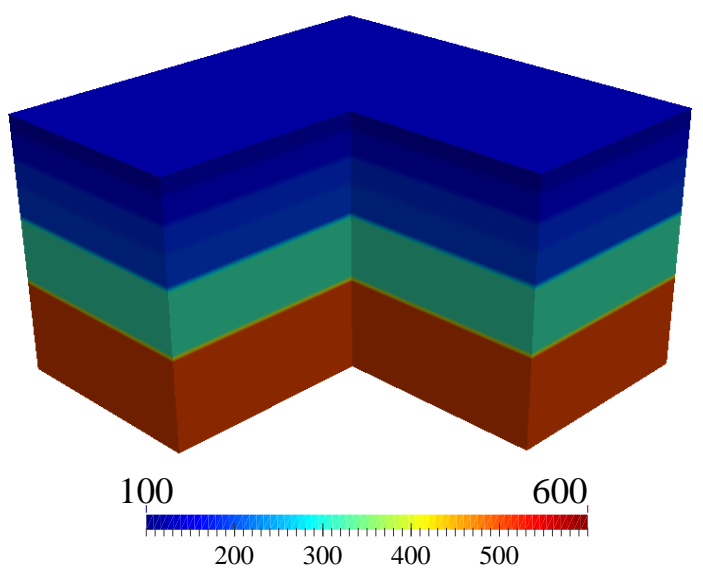

Figure 17: Inverted profile for $c_{s}$ via the SASW method.

Time-history comparisons at the 5 control sensors $s p_{1}-s p_{5}$ are shown in Fig. 18. These sensors are placed along the $x=0,10,30,40,60 \mathrm{~m}$ array lines. Overall, there is very good agreement between the measured field response and the response computed based on using the full-waveform inversion profiles. Specifically, the agreement at sensors $s p_{1}, s p_{2}, s p_{3}$ and $s p_{5}$ is remarkable. At $s p_{4}$, the amplitudes are not well-matched; moreover, the measured response and full-waveform-inversion-based response are out of phase after, approximately, $1.5 \mathrm{~s}$. As it can also be seen, the time-history response at the control sensors computed based on the SASW profiles, differ from the recorded response: overall, it appears that the SASW-based traces match the frequency content of the measurements, but not the amplitude.

Time-history comparisons for the remaining 30 sensors (see Fig. 9) are shown in Figs. 1923. Time history traces corresponding to the computed response based on the SASWinverted profiles are included for the first 6 sensors only (Fig. 19). As it can be seen, the SASW traces depart from the recorded response; this trend is true for the remaining 24 sensors too. We remark that it may not be fair to compare the SASW-based time histories at these locations with those resulting from the full-waveform inversion: these sensors were 


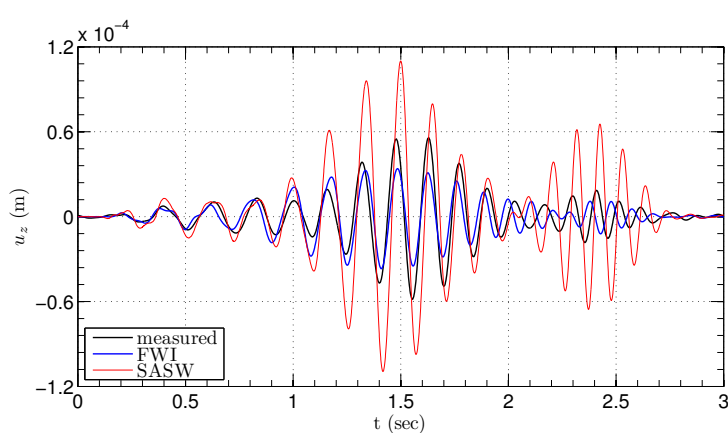

(a) $s p_{1}:(x, y)=(0,10) \mathrm{m}$

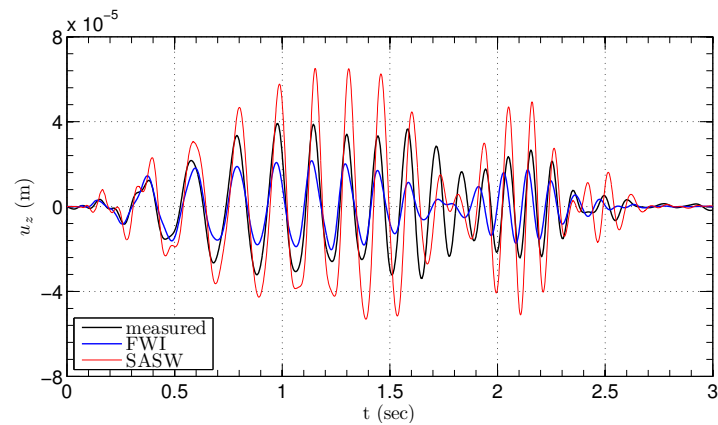

(c) $s p_{3}:(x, y)=(30,50) \mathrm{m}$

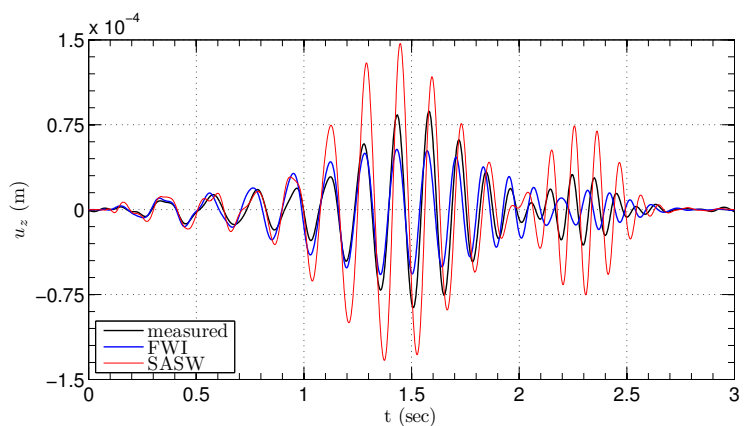

(b) $s p_{2}:(x, y)=(10,40) \mathrm{m}$

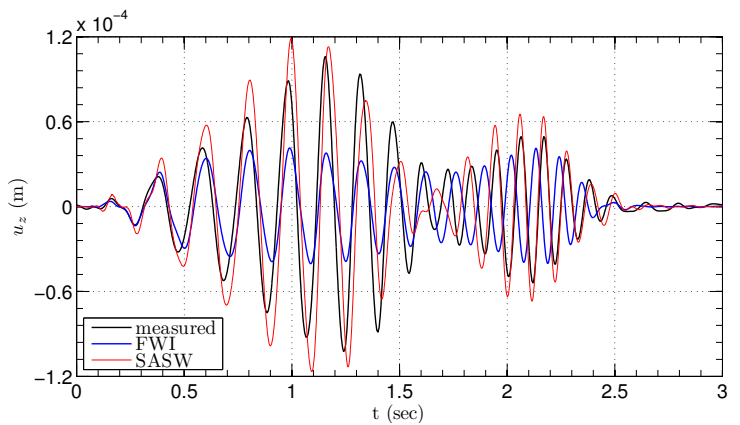

(d) $s p_{4}:(x, y)=(40,30) \mathrm{m}$

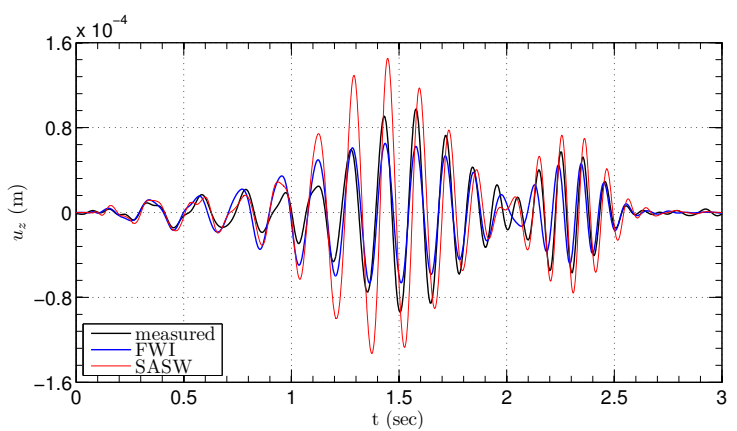

(e) $s p_{5}:(x, y)=(60,20) \mathrm{m}$

Figure 18: Comparison of measured surface displacement time-histories against those resulting from the SASW and full-waveform-inversion-based profiles at the control sensors (sensors not used for the fullwaveform inversion). 
used during the inversion process to minimize the misfit between the measured response and the computed response; therefore, good agreement between the full-waveform-inversionbased time-histories and the measured response is expected, as is indeed the case. Overall, the plots indicate that the optimizer performed successfully in matching the computed response with the measured response.

\subsection{Groundwater level effect}

According to prior investigations conducted at the site, the groundwater table is estimated to be between $6 \mathrm{~m}$ and $20 \mathrm{~m}$. While one would have expected that in fully saturated zones, the $\mathrm{P}$-wave velocity would take values closer to $1500 \mathrm{~m} / \mathrm{s}$, the full-waveform inversion did not return a $c_{p}$ profile suggestive of saturation at any depth up to $40 \mathrm{~m}$. Thus, in this section, we investigate the sensitivity of the computed response to variations of the groundwater level. To this end, we perform two forward wave simulations: one that corresponds to a groundwater level at $z=-6 \mathrm{~m}$, henceforth denoted by FWI-6, and another one, FWI-20, which corresponds to a groundwater level at $z=-20 \mathrm{~m}$. We compare the computed responses corresponding to FWI, FWI-6, and FWI-20, against the measured response. In these simulations, we use the full-waveform-inversion-based $c_{s}$ profile. Moreover, for soil depths above the water table, we use the $c_{p}$ profile that had been obtained earlier from full-waveform inversion; by contrast, for soil depths below the water table, we set $c_{p}=1500 \mathrm{~m} / \mathrm{s}$ uniformly. The resulting time history comparisons at the 5 control sensors $s p_{1}-s p_{5}$ are shown in Fig. 24. It can be seen that the time-histories are not very sensitive to the precise location of the groundwater level for the considered cases. In Fig. 25, we compare the time histories for sensors located at $x=0 \mathrm{~m}$, where a conclusion similar to the above can be drawn. The response at the remaining sensors (not shown) follow a similar trend.

In summary, the elastodynamic model at the heart of the full-waveform-inversion does not account for saturation, and cannot reveal the groundwater level, as the sensitivity results suggest. This limitation is also present in most other site characterization approaches. We remark that overcoming the limitation is possible within the FWI framework by appropriate modifications. Such an enhancement will be addressed in a future communication.

\section{Conclusions}

We discussed recent progress in three-dimensional site characterization using full-waveform inversion. We reviewed key components required for developing a general and robust framework to image the three-dimensional P- and S-wave velocity profiles of near-surface deposits. These components are: a) a computational model for the simulation of elastic waves in domains terminated by PMLs; b) a PDE-constrained optimization framework, endowed with regularization schemes, through which the computed response is "matched" to the measured response, via iteratively updating an initially assumed material distribution for the soil; and c) guidelines for designing a field experiment that can be well-coupled 


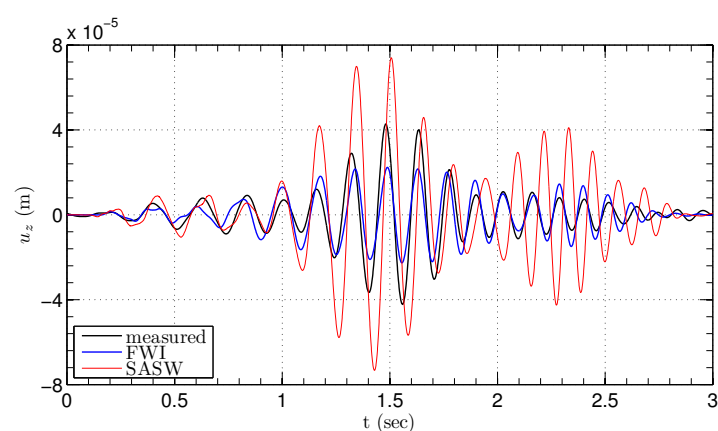

(a) $y=+60 \mathrm{~m}$

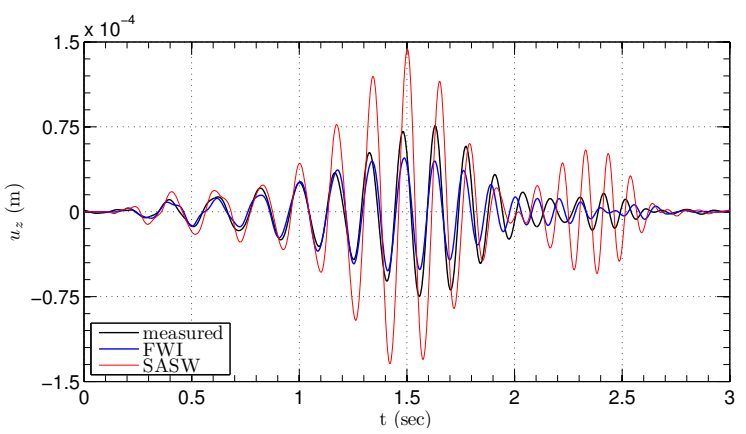

(c) $y=+40 \mathrm{~m}$

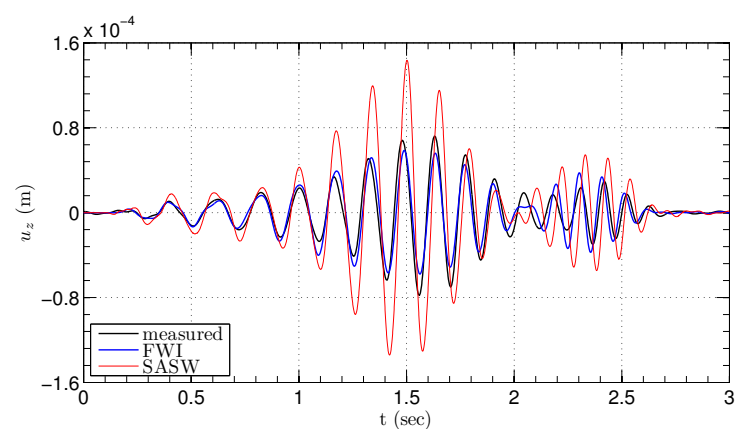

(e) $y=+20 \mathrm{~m}$

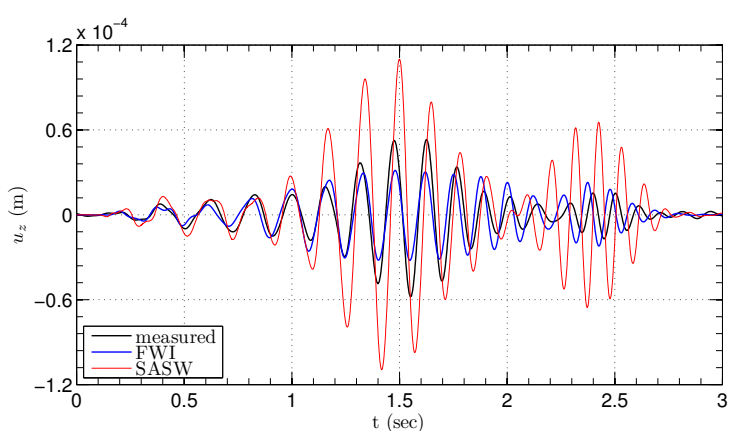

(b) $y=+50 \mathrm{~m}$

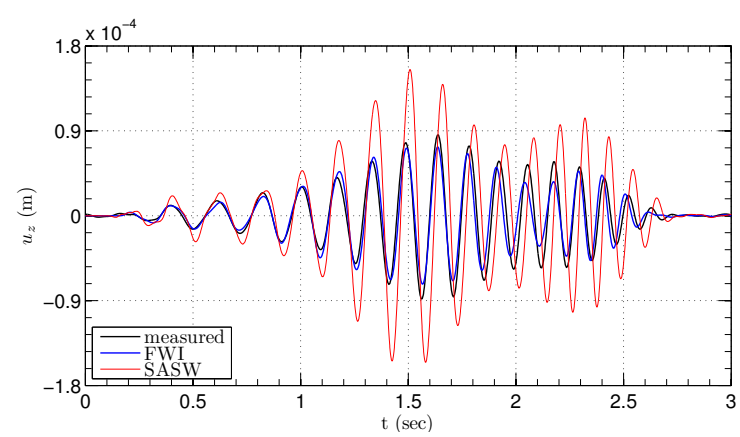

(d) $y=+30 \mathrm{~m}$

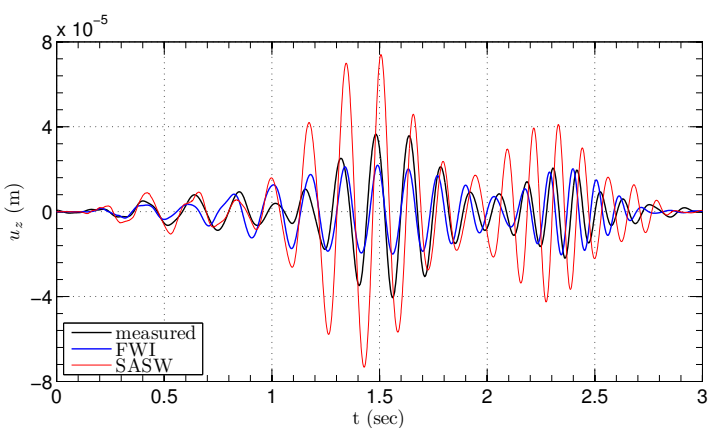

(f) $y=0 \mathrm{~m}$

Figure 19: Comparison of measured surface displacement time-histories against those resulting from the full-waveform-based inversion and SASW $(x=0 \mathrm{~m})$. 


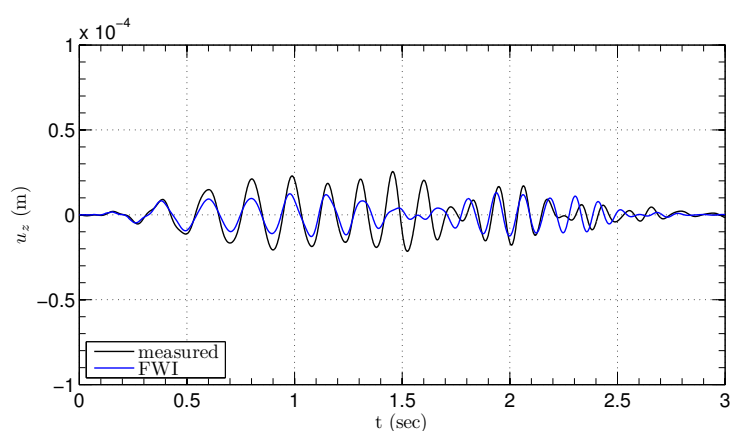

(a) $y=+60 \mathrm{~m}$

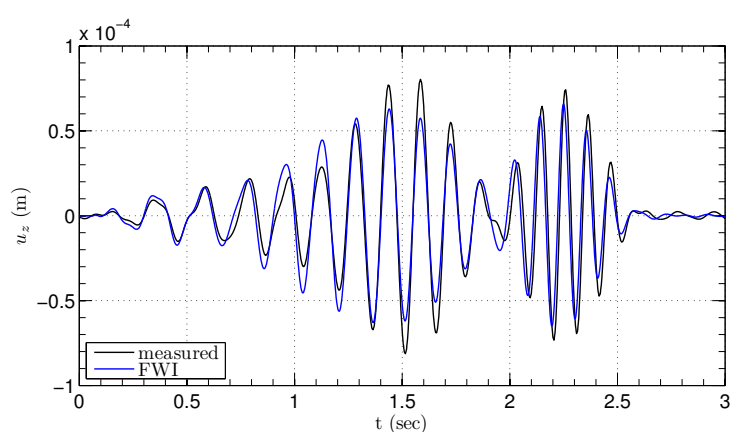

(c) $y=+30 \mathrm{~m}$

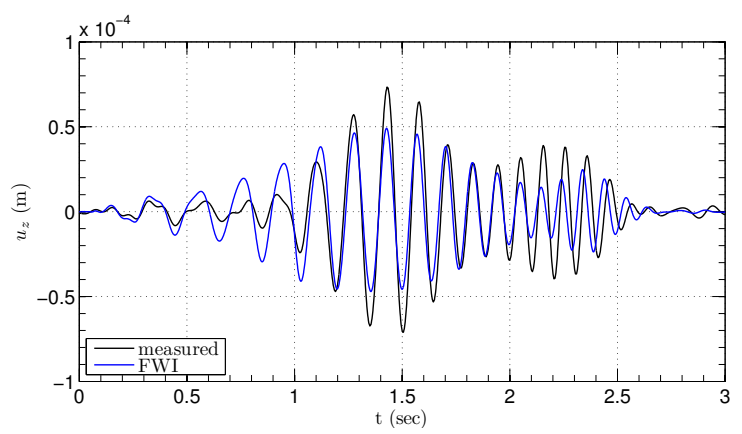

(e) $y=+10 \mathrm{~m}$

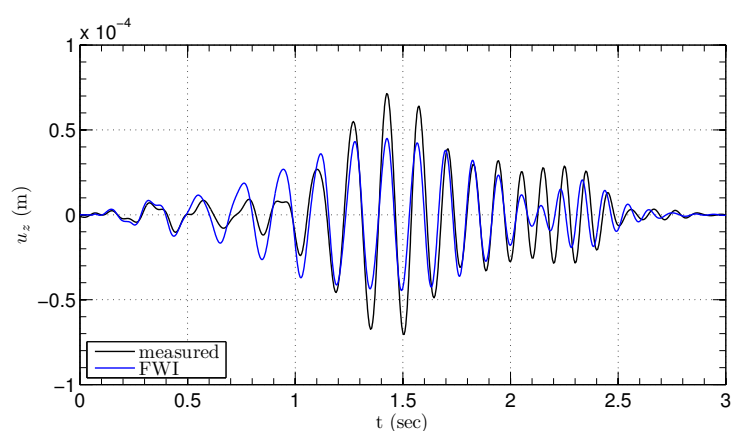

(b) $y=+50 \mathrm{~m}$

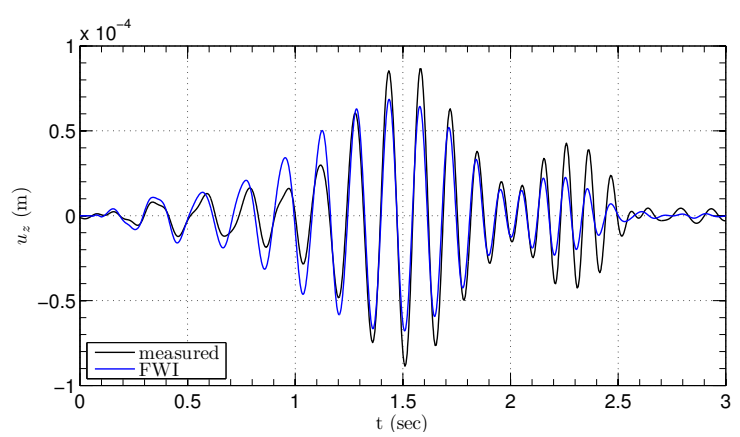

(d) $y=+20 \mathrm{~m}$

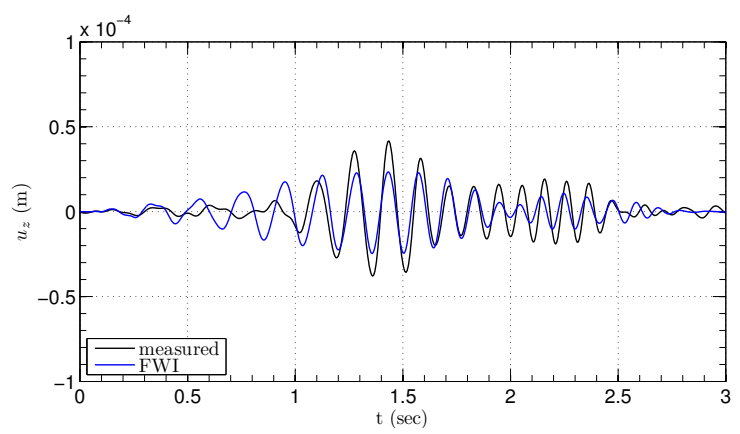

(f) $y=0 \mathrm{~m}$

Figure 20: Comparison of measured surface displacement time-histories against those resulting from the full-waveform-based inversion $(x=+10 \mathrm{~m})$. 


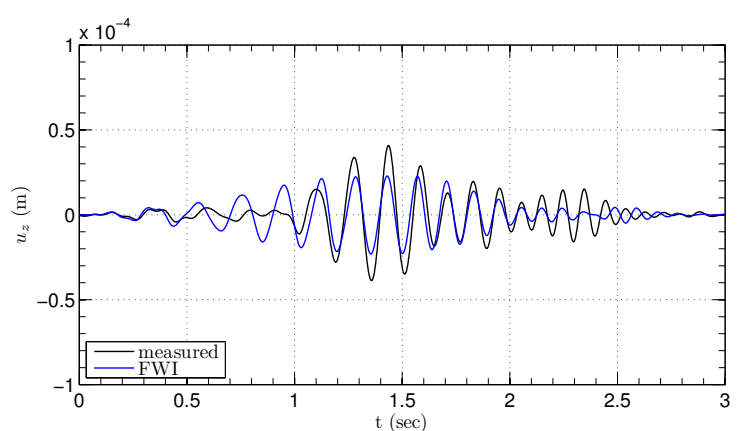

(a) $y=+60 \mathrm{~m}$

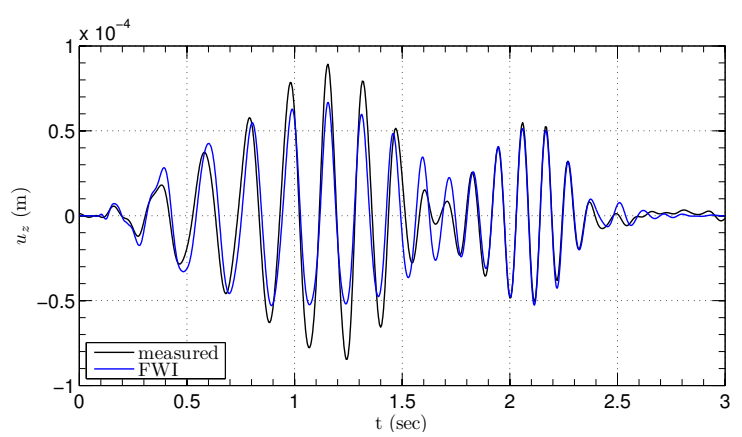

(c) $y=+30 \mathrm{~m}$

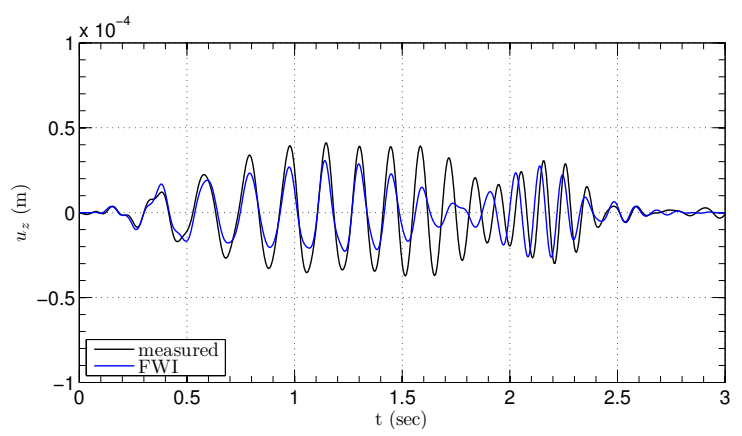

(e) $y=+10 \mathrm{~m}$

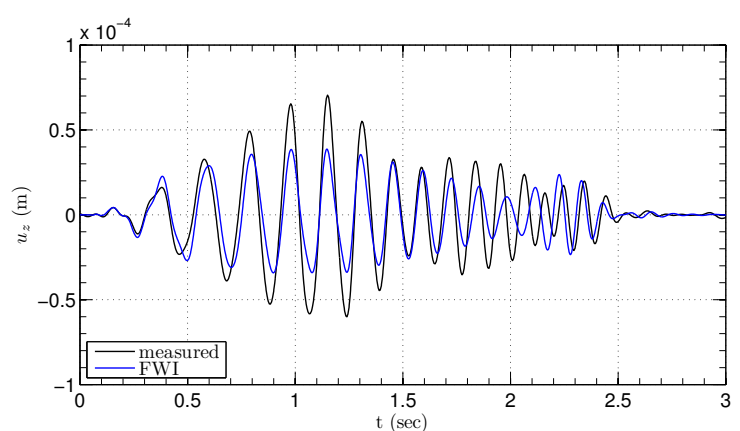

(b) $y=+40 \mathrm{~m}$

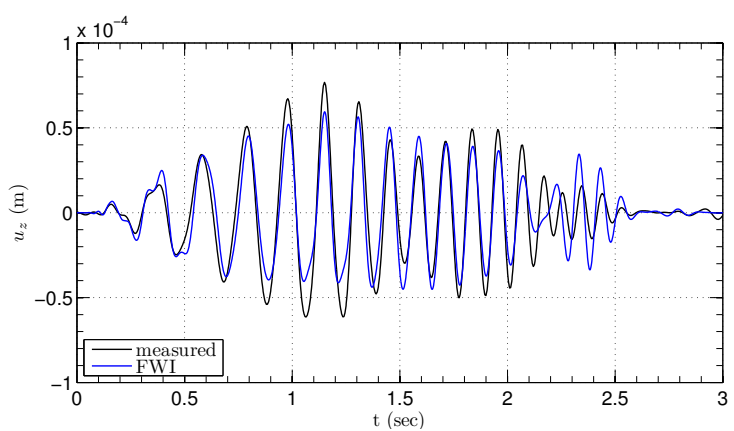

(d) $y=+20 \mathrm{~m}$

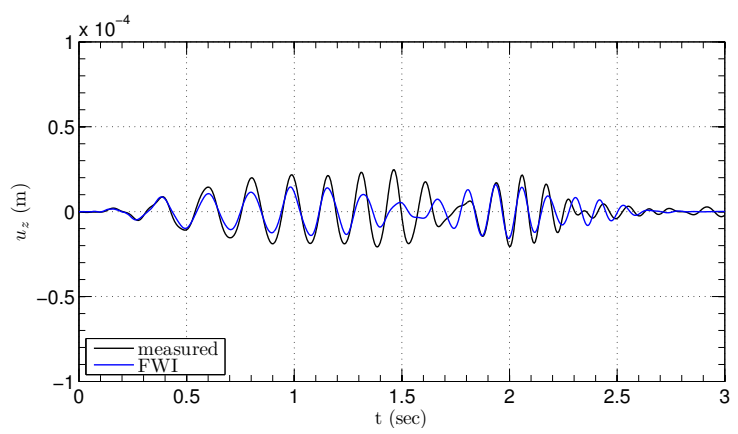

(f) $y=0 \mathrm{~m}$

Figure 21: Comparison of measured surface displacement time-histories against those resulting from the full-waveform-based inversion $(x=+30 \mathrm{~m})$. 


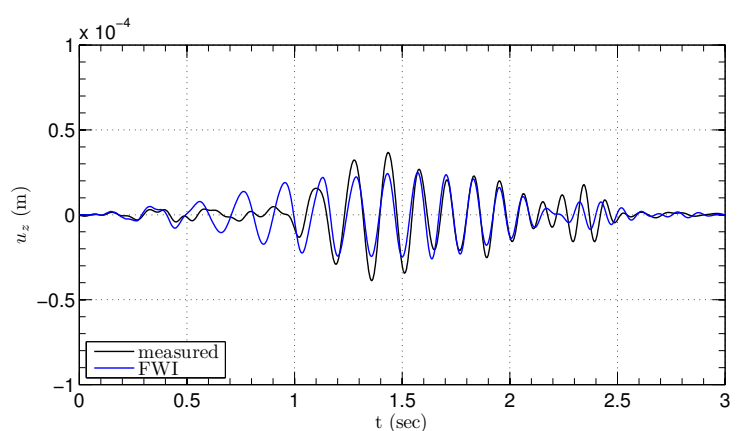

(a) $y=+60 \mathrm{~m}$

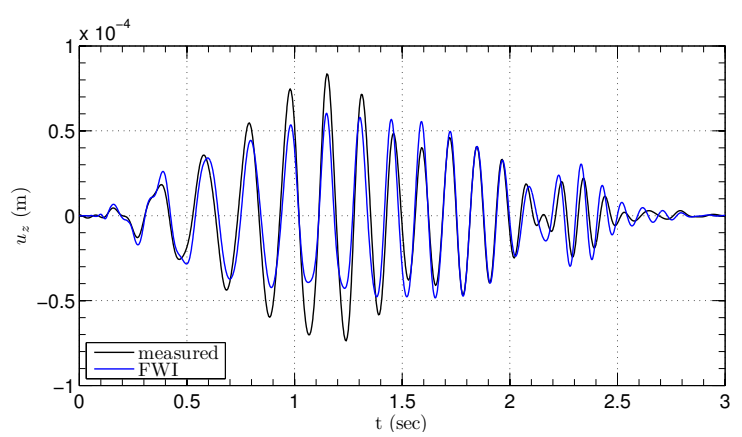

(c) $y=+40 \mathrm{~m}$

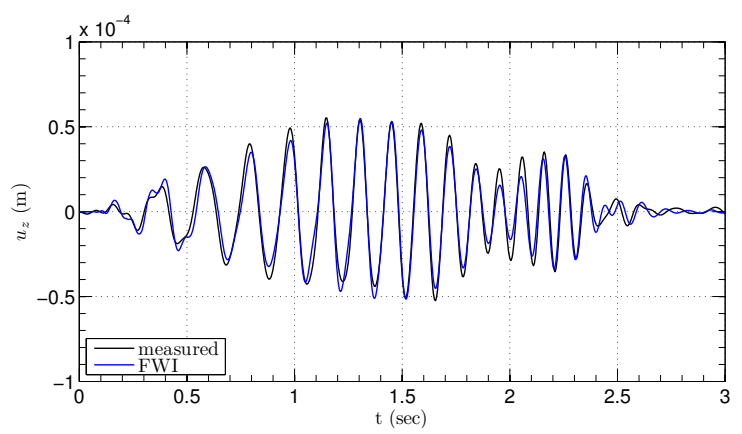

(e) $y=+10 \mathrm{~m}$

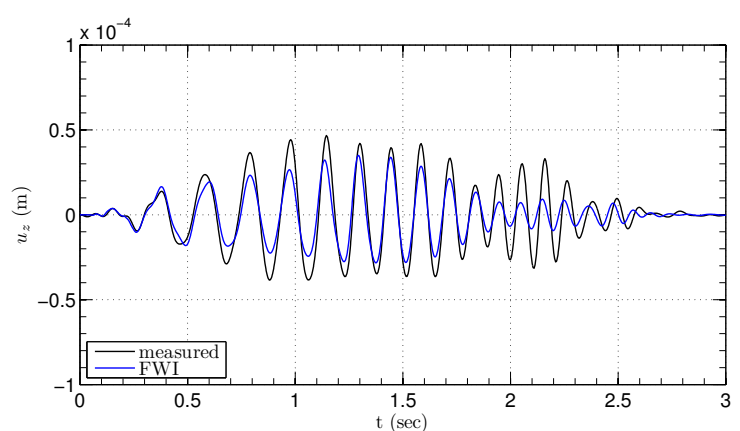

(b) $y=+50 \mathrm{~m}$

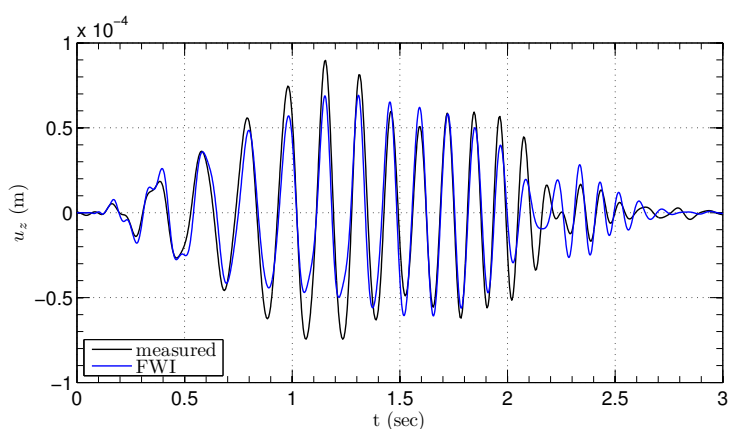

(d) $y=+20 \mathrm{~m}$

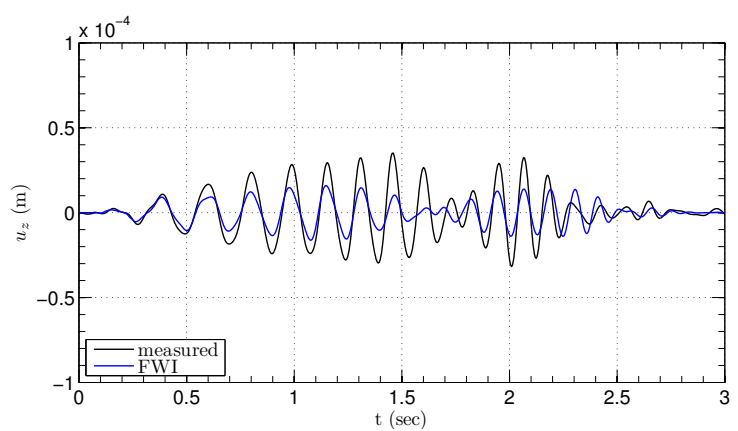

(f) $y=0 \mathrm{~m}$

Figure 22: Comparison of measured surface displacement time-histories against those resulting from the full-waveform-based inversion $(x=+40 \mathrm{~m})$. 


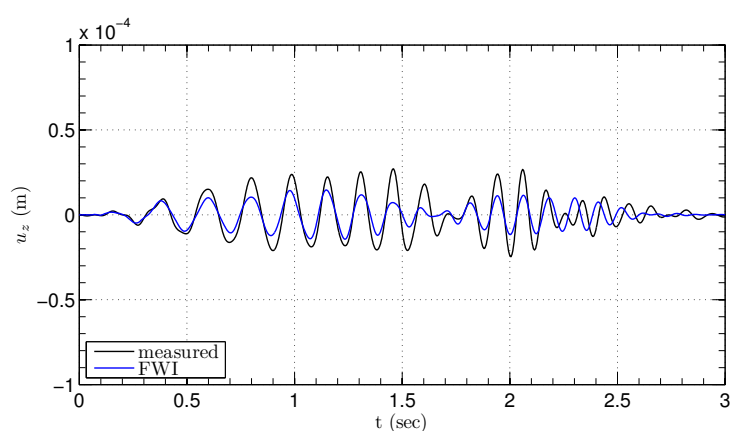

(a) $y=+60 \mathrm{~m}$

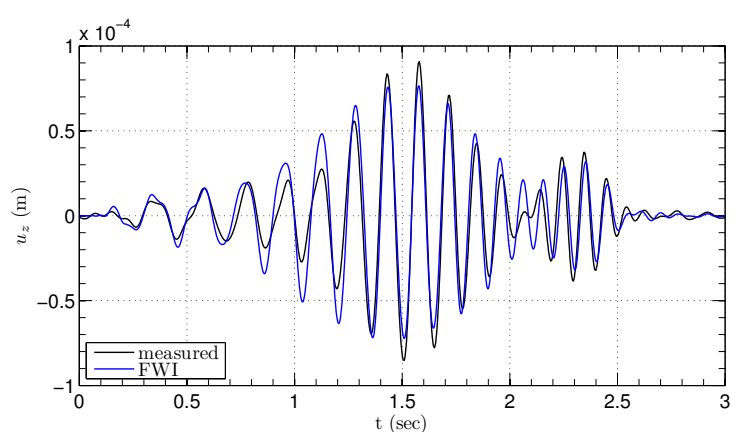

(c) $y=+40 \mathrm{~m}$

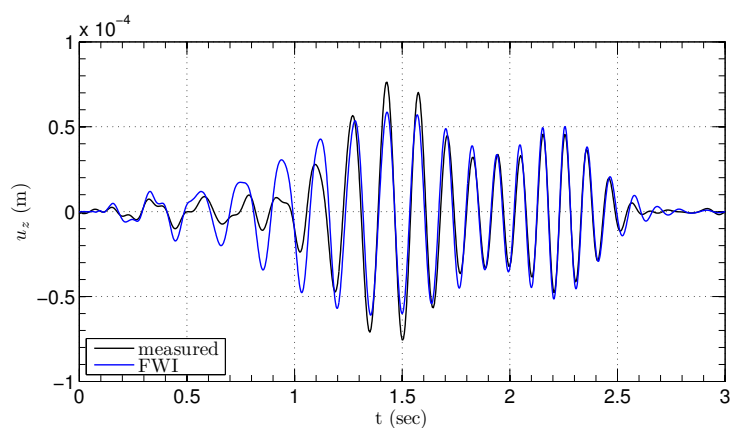

(e) $y=+10 \mathrm{~m}$

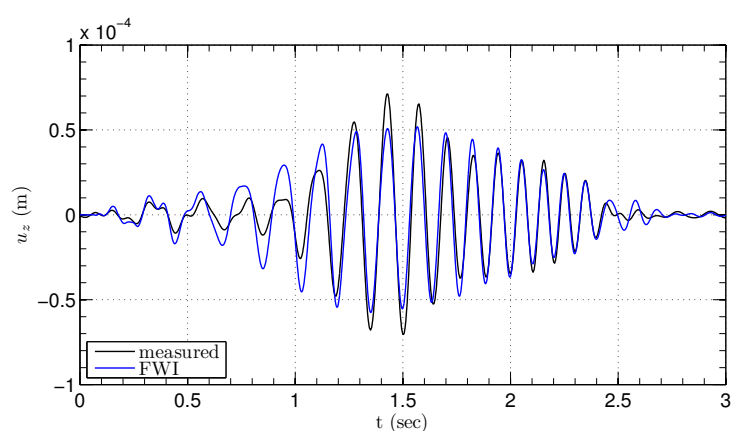

(b) $y=+50 \mathrm{~m}$

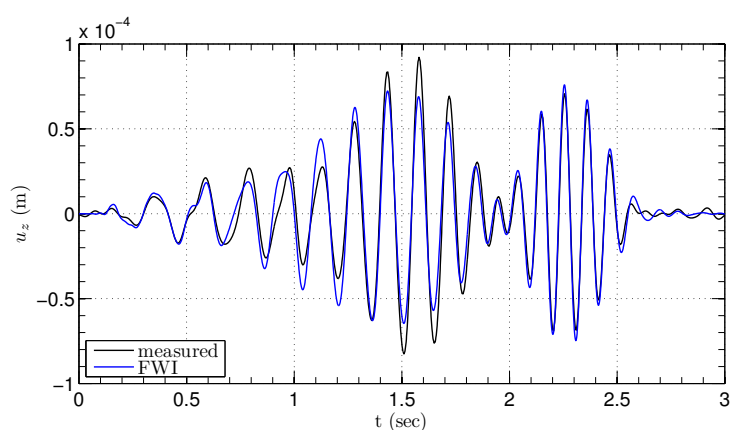

(d) $y=+30 \mathrm{~m}$

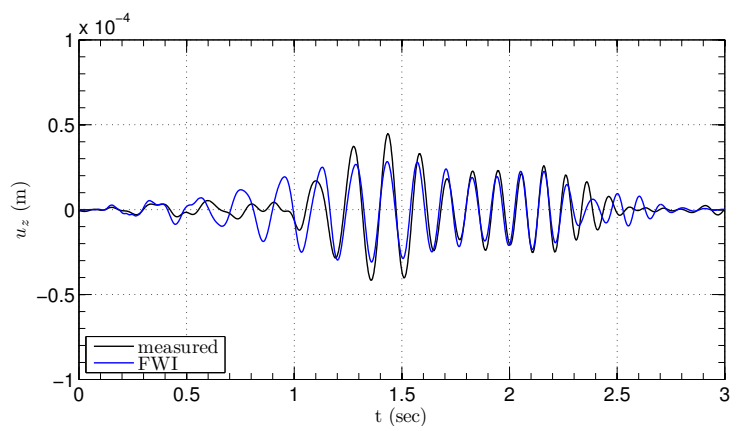

(f) $y=0 \mathrm{~m}$

Figure 23: Comparison of measured surface displacement time-histories against those resulting from the full-waveform-based inversion $(x=+60 \mathrm{~m})$. 


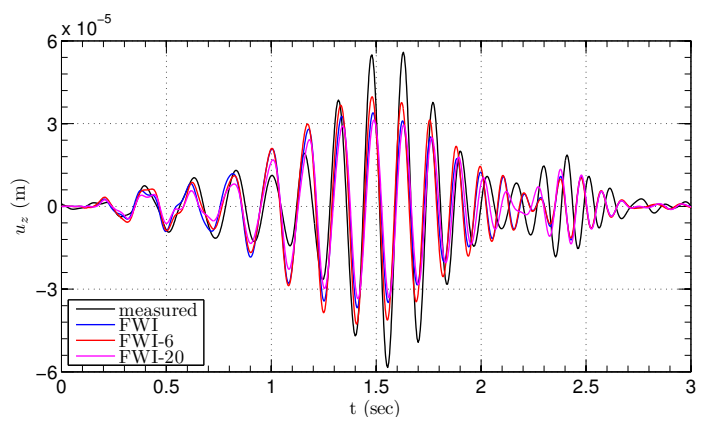

(a) $s p_{1}:(x, y)=(0,10) \mathrm{m}$

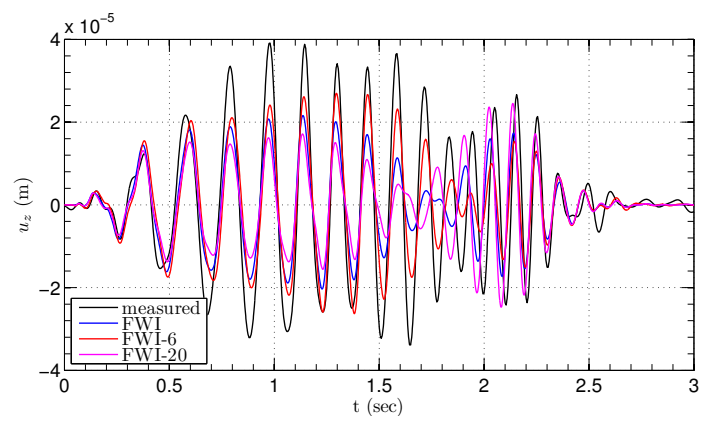

(c) $s p_{3}:(x, y)=(30,50) \mathrm{m}$

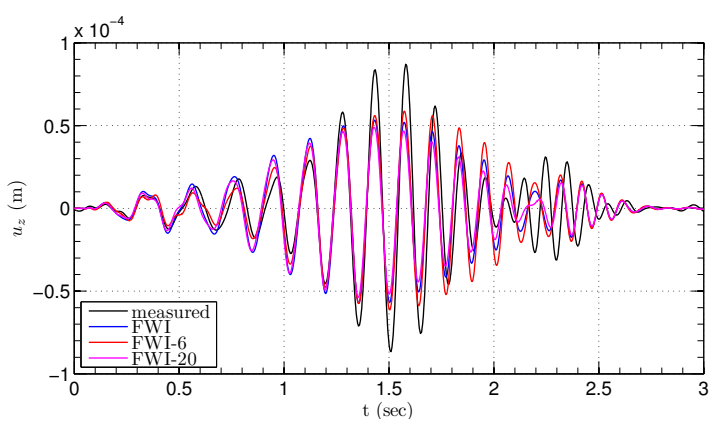

(b) $s p_{2}:(x, y)=(10,40) \mathrm{m}$

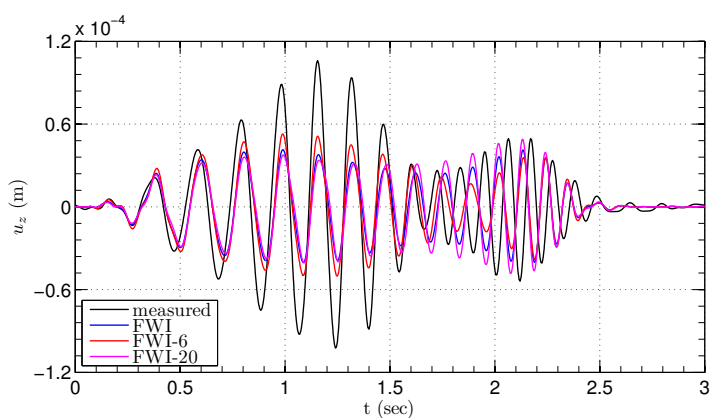

(d) $s p_{4}:(x, y)=(40,30) \mathrm{m}$

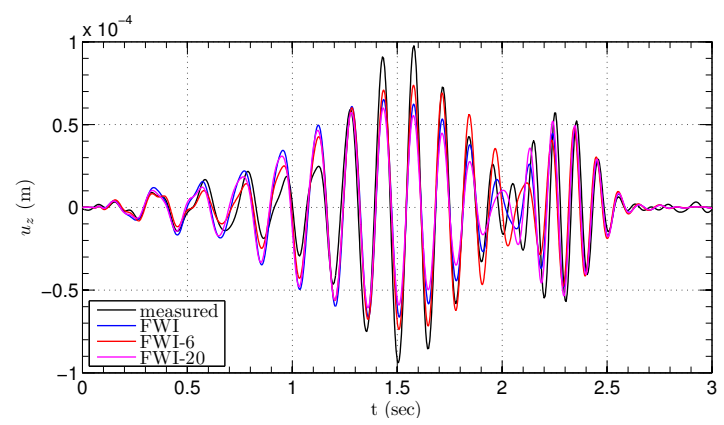

(e) $s p_{5}:(x, y)=(60,20) \mathrm{m}$

Figure 24: Comparison of measured surface displacement time-histories against those resulting from the fullwaveform-inversion-based profiles, with the groundwater level set at $6 \mathrm{~m}$ (FWI-6), at 20-m (FWI-20), and at infinity (FWI). The response is evaluated at the control sensors (sensors not used for the full-waveform inversion). 


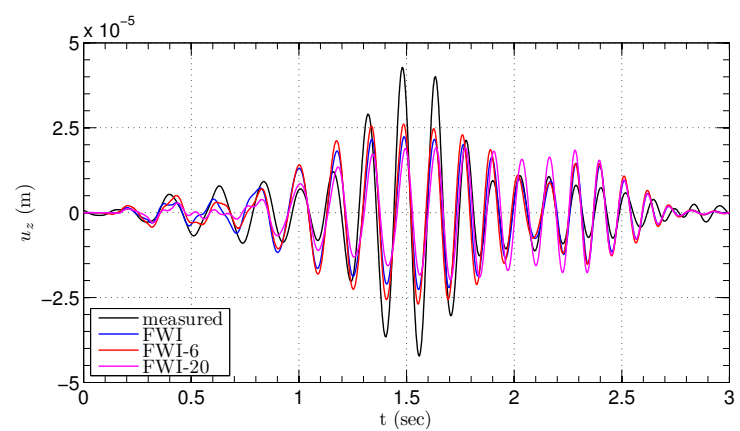

(a) $y=+60 \mathrm{~m}$

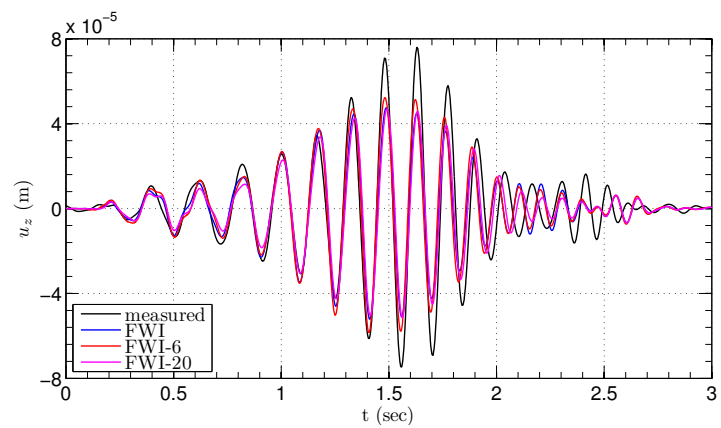

(c) $y=+40 \mathrm{~m}$

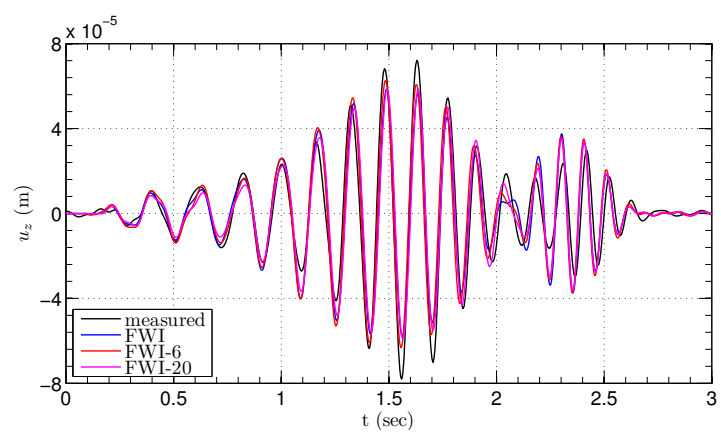

(e) $y=+20 \mathrm{~m}$

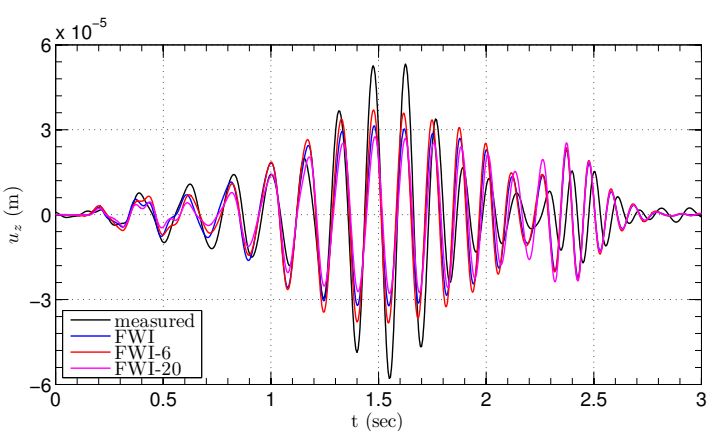

(b) $y=+50 \mathrm{~m}$

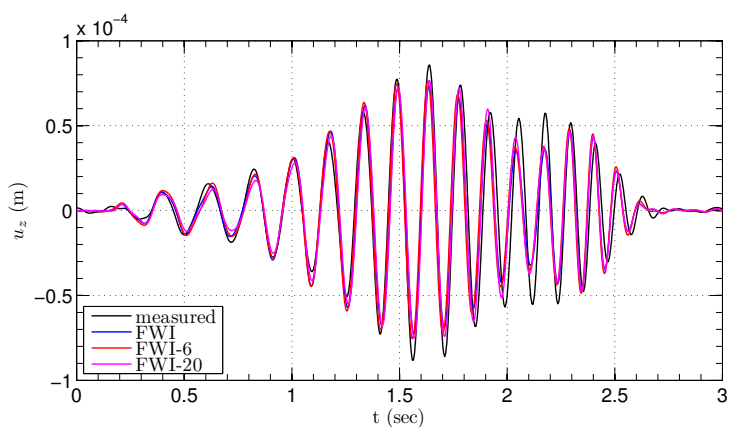

(d) $y=+30 \mathrm{~m}$

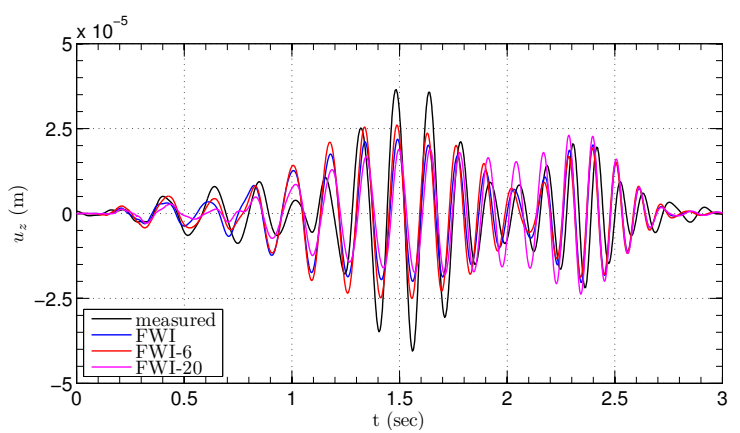

(f) $y=0 \mathrm{~m}$

Figure 25: Comparison of measured surface displacement time-histories against those resulting from the full-waveform-inversion-based profiles, with the groundwater level set at $6 \mathrm{~m}$ (FWI-6), at 20-m (FWI-20), and at infinity (FWI). The response is evaluated at the array line $x=0 \mathrm{~m}$. 
with the algorithmic development. We then reported on the imaging of the NEES@UCSB site in Garner Valley, using the reviewed computational framework. We compared the resulting imaging against the profiling obtained from the SASW method, and reported general agreement.

To drive the inversion process, we used only a subset of the sensors that we had deployed during the field experiment. In an attempt to validate the resulting profiles, we then used the remaining sensors as controls for time-history comparisons between the measured response, and a computed response based on either the full-waveform-inversion- or SASWbased profiles. The reported agreement at the control sensors was remarkably good, which, when considered in tandem with the excellent agreement of the time histories between computed and measured responses at the rest of the sensors, attests to the validity of the full-waveform-inversion-based profiles.

To the best of our knowledge, this is the first attempt that field data have been successfully used for three-dimensional P- and S-wave velocity full-waveform-based profiling of near-surface deposits, where PMLs have been used for the rigorous treatment of the truncation boundaries. Overall, the full-waveform inversion methodology discussed herein, appears robust, general, and promising for the imaging of near-surface deposits, in support of geotechnical site characterization investigations.

\section{Acknowledgments}

We wish to thank Dr. Jamison Steidl, Dr. Farn-Yuh Menq, Mr. Paul Hegarty, Mr. David Umberg, Dr. Y.-C. Lin, Ms. Julia Roberts, and Mr. Cecil Hoffpauir for assisting with conducting the field experiments and processing the SASW tests.

Partial support for the authors' research has been provided by the National Science Foundation under grant awards CMMI-0619078 and CMMI-1030728, and through an Academic Alliance Excellence grant between the King Abdullah University of Science and Technology in Saudi Arabia (KAUST) and the University of Texas at Austin. Support for operation of the NEES@UCSB and NEES@UTexas equipment sites was provided by the National Science Foundation grant awards CMMI-0927178 and CMMI-0927178, respectively. This support is gratefully acknowledged.

\section{Appendix A. Gradient of a functional}

The gradient of a functional $\mathcal{F}: \mathcal{H} \rightarrow \mathbb{R}$, where $\mathcal{H}$ is a Hilbert space, is defined as the Riesz-representation of the derivative $\mathcal{F}^{\prime}(\mathbf{q})(\tilde{\mathbf{q}})$, such that

$$
(\mathcal{G}(\mathbf{q}), \tilde{\mathbf{q}})_{\mathcal{H}}=\mathcal{F}^{\prime}(\mathbf{q})(\tilde{\mathbf{q}}) \quad \forall \tilde{\mathbf{q}} \in \mathcal{H},
$$

where $\mathcal{G}$ denotes the gradient, and we use the following notation for the Gâteaux derivative of $\mathcal{F}$ with respect to $\mathbf{q}$ in a direction $\tilde{\mathbf{q}}$ : 


$$
\mathcal{F}^{\prime}(\mathbf{q})(\tilde{\mathbf{q}})=\lim _{h \rightarrow 0} \frac{\mathcal{F}(\mathbf{q}+h \tilde{\mathbf{q}})-\mathcal{F}(\mathbf{q})}{h}
$$

\section{References}

[1] Garner Valley Downhole Array. http://nees.ucsb.edu/facilities/GVDA.

[2] U. Albocher, P. E. Barbone, M. S. Richards, A. A. Oberai, and I. Harari. Approaches to accommodate noisy data in the direct solution of inverse problems in incompressible plane strain elasticity. Inverse Problems in Science and Engineering, 22(8):1307-1328, 2014 .

[3] M. Amrouche and H. Yamanaka. Two-dimensional shallow soil profiling using timedomain waveform inversion. Geophysics, 80(1):EN27-EN41, 2015.

[4] B. Banerjee, T. F. Walsh, W. Aquino, and M. Bonnet. Large scale parameter estimation problems in frequency-domain elastodynamics using an error in constitutive equation functional. Comp. Meth. Appl. Mech. Eng., 253:60-72, 2013.

[5] U. Basu. Explicit finite element perfectly matched layer for transient three-dimensional elastic waves. Int. J. Numer. Meth. Engng., 77:151-176, 2009.

[6] J. Bielak, F. Civilini, J. Crempien, A. Fathi, R. Gee, P. Hegarty, L.F. Kallivokas, F.-Y. Menq, J. Steidl, K.H. Stokoe, and D. Umberg. T-Rex shaking at Garner Valley: toward three-dimensional full-waveform inversion, 2013. doi:10.4231/D3BK16P79.

[7] E. Bozda, J. Trampert, and J. Tromp. Misfit functions for full waveform inversion based on instantaneous phase and envelope measurements. Geophysical Journal International, 185(2):845-870, 2011.

[8] C. Bunks, F. Saleck, S. Zaleski, and G. Chavent. Multiscale seismic waveform inversion. Geophysics, 60(5):1457-1473, 1995.

[9] M. I. Diaz, W. Aquino, and M. Bonnet. A modified error in constitutive equation approach for frequency-domain viscoelasticity imaging using interior data. Computer Methods in Applied Mechanics and Engineering, 296:129 - 149, 2015.

[10] B. Engquist and B. Froese. Application of the Wasserstein metric to seismic signals. Communications in Mathematical Science, 12(5):979-988, 2014.

[11] I. Epanomeritakis, V. Akcelik, O. Ghattas, and J. Bielak. A Newton-CG method for large-scale three-dimensional elastic full-waveform seismic inversion. Inverse Problems, 24(3):034015, 2008. 
[12] M. Eslaminia and M.N. Guddati. A novel wave equation solver to increase the efficiency of full waveform inversion. SEG Technical Program Expanded Abstracts, pages 1028-1032, 2014.

[13] A. Fathi. Full-waveform inversion in three-dimensional PML-truncated elastic media: theory, computations, and field experiments. Ph.D. thesis, The University of Texas at Austin, 2015.

[14] A. Fathi, L. F. Kallivokas, and B. Poursartip. Full-waveform inversion in threedimensional PML-truncated elastic media. Computer Methods in Applied Mechanics and Engineering, 296:39 - 72, 2015.

[15] A. Fathi, B. Poursartip, and L. F. Kallivokas. Time-domain hybrid formulations for wave simulations in three-dimensional PML-truncated heterogeneous media. International Journal for Numerical Methods in Engineering, 101(3):165-198, 2015.

[16] A. Fichtner, J. Trampert, P. Cupillard, E. Saygin, T. Taymaz, Y. Capdeville, and A. Villaseor. Multiscale full waveform inversion. Geophysical Journal International, 194(1):534-556, 2013.

[17] A. Gholami, A. Mang, and G Biros. An inverse problem formulation for parameter estimation of a reaction-diffusion model of low grade gliomas. Journal of Mathematical Biology, pages 1-25, 2015.

[18] S. H. Joh. Advances in the data interpretation technique for spectral-analysis-ofsurface-waves $(S A S W)$ measurements. Ph.D. thesis, The University of Texas at Austin, 1996.

[19] L.F. Kallivokas, A. Fathi, S. Kucukcoban, K.H. Stokoe II, J. Bielak, and O. Ghattas. Site characterization using full waveform inversion. Soil Dynamics and Earthquake Engineering, 47:62 - 82, 2013.

[20] J. W. Kang and L. F. Kallivokas. The inverse medium problem in heterogeneous PML-truncated domains using scalar probing waves. Comput. Methods Appl. Mech. Engrg., 200:265-283, 2011.

[21] S. Kucukcoban. The inverse medium problem in PML-truncated elastic media. Ph.D. thesis, The University of Texas at Austin, 2010.

[22] V. Monteiller, S. Chevrot, D. Komatitsch, and N. Fuji. A hybrid method to compute short-period synthetic seismograms of teleseismic body waves in a 3-D regional model. Geophysical Journal International, 192(1):230-247, 2013.

[23] V. Monteiller, S. Chevrot, D. Komatitsch, and Y. Wang. Three-dimensional full waveform inversion of short-period teleseismic wavefields based upon the SEM-DSM hybrid method. Geophysical Journal International, 202(2):811-827, 2015. 
[24] P. Mora. Nonlinear two-dimensional elastic inversion of multioffset seismic data. Geophysics, 52(9):1211-1228, 1987.

[25] S.-W. Na and L. F. Kallivokas. Direct time-domain soil profile reconstruction for one-dimensional semi-infinite domains. Soil Dynamics and Earthquake Engineering, 29:1016-1026, 2009.

[26] J. Nocedal and S. Wright. Numerical Optimization. Springer Series in Operations Research. Springer, New York, NY, second edition, 2006.

[27] A. A. Oberai, N. H. Gokhale, M. M. Doyley, and M. Bamber. Evaluation of the adjoint equation based algorithm for elasticity imaging. Physics in Medicine and Biology, 49:2955-2974, 2004.

[28] A. A. Oberai, N. H. Gokhale, and G. R. Feijo. Solution of inverse problems in elasticity imaging using the adjoint method. Inverse Problems, 19(2):297-313, 2003.

[29] A. Pakravan, J. W. Kang, and C.M. Newtson. A Gauss-Newton full-waveform inversion for material profile reconstruction in viscoelastic semi-infinite solid media. Inverse Problems in Science and Engineering, doi:10.1080/17415977.2015.1046861, 2015.

[30] C. B. Park, R. D. Miller, and J. Xia. Multichannel analysis of surface waves. Geophysics, 64:800-808, 1999.

[31] N. Petra and G. Stadler. Model variational inverse problems governed by partial differential equations. ICES Report 11-05, The Institute for Computational Engineering and Sciences, The University of Texas at Austin, 2011.

[32] J. G. Proakis and D. K. Manolakis. Digital Signal Processing. Prentice Hall, Englewood Cliffs, NJ, fourth edition, 2006.

[33] K. H. Stokoe II, S. G. Wright, J. A. Bay, and J. M. Roësset. Characterization of geotechnical sites by SASW method. In R. D. Woods, editor, Geophysical Characterization of Sites, pages 15-25. Oxford \& IBH Pub. Co., New Delhi, India, 1994.

[34] A. Tikhonov. Solution of incorrectly formulated problems and the regularization method. Soviet Math. Dokl., 5:1035/1038, 1963.

[35] P. Tong, C. W. Chen, D. Komatitsch, P. Basini, and Q. Liu. High-resolution seismic array imaging based on an SEM-FK hybrid method. Geophysical Journal International, 197(1):369-395, 2014.

[36] K. T. Tran and M. McVay. Site characterization using Gauss-Newton inversion of 2-D full seismic waveform in the time domain. Soil Dynamics and Earthquake Engineering, $43: 16-24,2012$. 
[37] F. Tröltzsch. Optimal Control of Partial Differential Equations: Theory, Methods, and Applications, volume 112 of Graduate Studies in Mathematics. American Mathematical Society, 2010.

[38] C. R. Vogel. Computational Methods for Inverse Problems. Frontiers in Applied Mathematics. Society for Industrial and Applied Mathematics (SIAM), Philadelphia, PA, 2002.

[39] Z. Xie, D. Komatitsch, R. Martin, and R. Matzen. Improved forward wave propagation and adjoint-based sensitivity kernel calculations using a numerically stable finite-element PML. Geophysical Journal International, 198(3):1714-1747, 2014. 